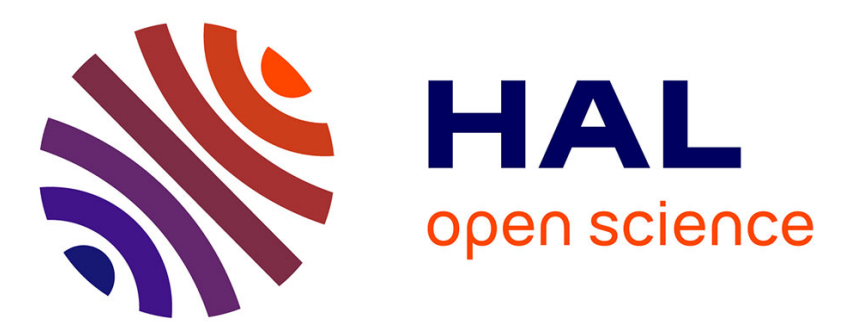

\title{
Distortion of DNA cholesteric liquid crystal quenched at low temperature: geometrical analysis and models
}

\author{
A. Leforestier, F. Livolant
}

\section{To cite this version:}

A. Leforestier, F. Livolant. Distortion of DNA cholesteric liquid crystal quenched at low temperature: geometrical analysis and models. Journal de Physique II, 1992, 2 (10), pp.1853-1880. 10.1051/jp2:1992240 . jpa-00247772

\section{HAL Id: jpa-00247772 https://hal.science/jpa-00247772}

Submitted on 1 Jan 1992

HAL is a multi-disciplinary open access archive for the deposit and dissemination of scientific research documents, whether they are published or not. The documents may come from teaching and research institutions in France or abroad, or from public or private research centers.
L'archive ouverte pluridisciplinaire HAL, est destinée au dépôt et à la diffusion de documents scientifiques de niveau recherche, publiés ou non, émanant des établissements d'enseignement et de recherche français ou étrangers, des laboratoires publics ou privés. 
Classification

Physics Abstracts

$61.30-61.70-87.15$

\title{
Distortion of DNA cholesteric liquid crystal quenched at low temperature : geometrical analysis and models
}

\author{
A. Leforestier and F. Livolant \\ Centre de Biologie Cellulaire (CNRS), 67 rue Maurice Günsbourg, 94205 Ivry-sur-Seine Cedex, \\ France
}

(Received 5 March 1992, accepted in final form 16 July 1992)

\begin{abstract}
Résumé. - La microscopie électronique associée à la technique de cryofracture permet une analyse ultrastructurale des déformations de l'organisation cristalline liquide cholestérique d'un échantillon d'ADN en solution : lors de la congélation par projection contre un bloc de cuivre refroidi à environ $10 \mathrm{~K}$ par de l'hélium liquide, l'échantillon est soumis à des forces de compression parallèles à la stratification cholestérique. Nous présentons une modélisation géométrique de ces déformations. Après un bref rappel de la représentation schématique de la structure cholestérique par une série de plans fictifs, nous montrons que ces plans sont alors transformés en surfaces sinusoïdales dont la période est très inférieure au pas cholestérique et l'amplitude modulée en fonction de l'orientation relative des molécules et de la force de compression. Les conséquences de telles déformations sur l'émergence de double twist dans la structure sont examinées. Pour les plus faibles valeurs de concentration en ADN dans la phase cholestérique, nous observons des structures hélicoïdales complexes. Une relation avec le premier phénomène décrit est envisagée et discutée.
\end{abstract}

Abstract. - Freeze-fracture electron microscopy allows an ultrastructural analysis of deformations of a DNA cholesteric liquid crystalline sample subjected to a compressive stress applied parallel to the layers, when quickly frozen by projection onto a copper block cooled down to about $10 \mathrm{~K}$. A geometrical model of these deformations is proposed. After a brief recall of the usual representation of the cholesteric structure as a succession of equidistant pseudoplanes, we show that these planes are distorted into sinusoidal surfaces whose wavelength is much smaller than the cholesteric pitch and its amplitude modulated with the average molecular orientation relative to the compressive force. The consequences of these deformations regarding double twist occurrence within the structure are analysed. When the DNA concentration in the cholesteric mesophase is low, a complex helicoidal structure is observed. A relationship between these two phenomena is considered and discussed.

\section{Introduction.}

Electron microscopic analysis of liquid crystalline phases has recently aroused strong interest [1-12], because it directly provides structural data at the molecular level. The ultrastructural analysis of lyotropic aqueous mesophases raises the problem of the fixation of highly hydrated material without addition of any cryoprotectant. Quick-freezing by projection onto a copper 
block cooled down to liquid helium temperature was shown to prevent the formation of any visible ice crystals within the DNA cholesteric mesophase that contains more than $80 \%$ water [13]. Notwithstanding, the cholesteric patterns observed on freeze-fracture replicas of this material display multiple discrepancies regarding the regular cholesteric organization. It is possible to relate the deformation of this quenched cholesteric structure to our cryofixation method. Indeed, DNA samples frozen by immersion into a liquid cryogen instead of by squashing onto a cold metallic surface do never show deformations of the cholesteric organization (unpublished data). We can therefore assume that they are induced experimentally by the compressive stress undergone by the sample during its projection against the copper block. Since the quality of the replicas allows an accurate visualization of the molecular orientations and thus provides a precise local description of the molecular arrangements, our experiments allow the analysis of the structural instabilities that arise in cholesteric liquid crystals under a compressive stress, since they are quenched here before the structure relaxes. We model these deformations and present their geometrical analyses at the molecular level.

\section{Experimental.}

2.1 BIOLOGICAL MATERIAL. - DNA fragments of about $50 \mathrm{~nm}$ were prepared by selective digestion of calf thymus chromatin with micrococcal nuclease and characterized by electrophoresis on polyacrylamide gels as previously described by Strzelecka and Rill [14]. Purified DNA was dissolved in a $\mathrm{pH}=7$ buffer containing $0.25 \mathrm{M}$ ammonium acetate, $10 \mathrm{mM}$ sodium cacodylate, $0.5 \mathrm{mM}$ EDTA, at a concentration of about $200 \mathrm{mg} / \mathrm{ml}$. Under these conditions, DNA molecules form a liquid crystalline cholesteric phase which was checked in polarizing microscopy.

2.2 FREEZING PROCESS AND ELECTRON MICROSCOPY. - Cholesteric liquid crystalline samples were deposited onto copper discs (Fig. 1a). Part of the samples was stored for about one hour in a humidity chamber in order to stabilize at a given concentration, while others were subjected to slow evaporation of the solvent in order to obtain a concentration gradient. They were frozen quickly by projection (with a velocity of about $3 \mathrm{~m} . \mathrm{s}^{-1}$ ) onto a copper block cooled down to about $10 \mathrm{~K}$ by liquid helium, using a Reichert-Jung cryovacublock device (Fig. 1b) and immediately transferred into liquid nitrogen. The surface of the sample is flattened by this freezing process. This method was shown to lead to a cryofixation that avoids the formation of any visible ice crystal [13].

Frozen samples were placed in a Balzers BAF 400T apparatus and fractured within $10 \mu \mathrm{m}$ of the upper surface at $-110^{\circ} \mathrm{C}$ under a $2 \times 10^{-7}$ Torr vacuum with a razor blade parallel to the sample surface. The fracture surface, though parallel at the macroscopic level to the blade orientation, presents strong local variations, thus composing a set of variously oriented microscopic domains (Fig. 1c). The surface of the sample was unidirectionally shadowed with $20 \AA$ of platinum/carbon at an angle of $45^{\circ}$ and coated with a $200 \AA$ thick carbon layer. Replicas were washed in distilled water and observed in a 201 Philips TEM at $80 \mathrm{kV}$.

\section{Results.}

3.1 ChOlesteriC STRUCTURE IN ELECTRON MICROSCOPY. - Freeze-fracture electron microscopy of a cholesteric liquid crystalline phase reveals the three typical kinds of patterns that correspond to the different possible orientations of the fracture plane relative to the cholesteric axis $C$ [9, Leforestier \& Livolant, in preparation]. The fracture plane may be normal, parallel 


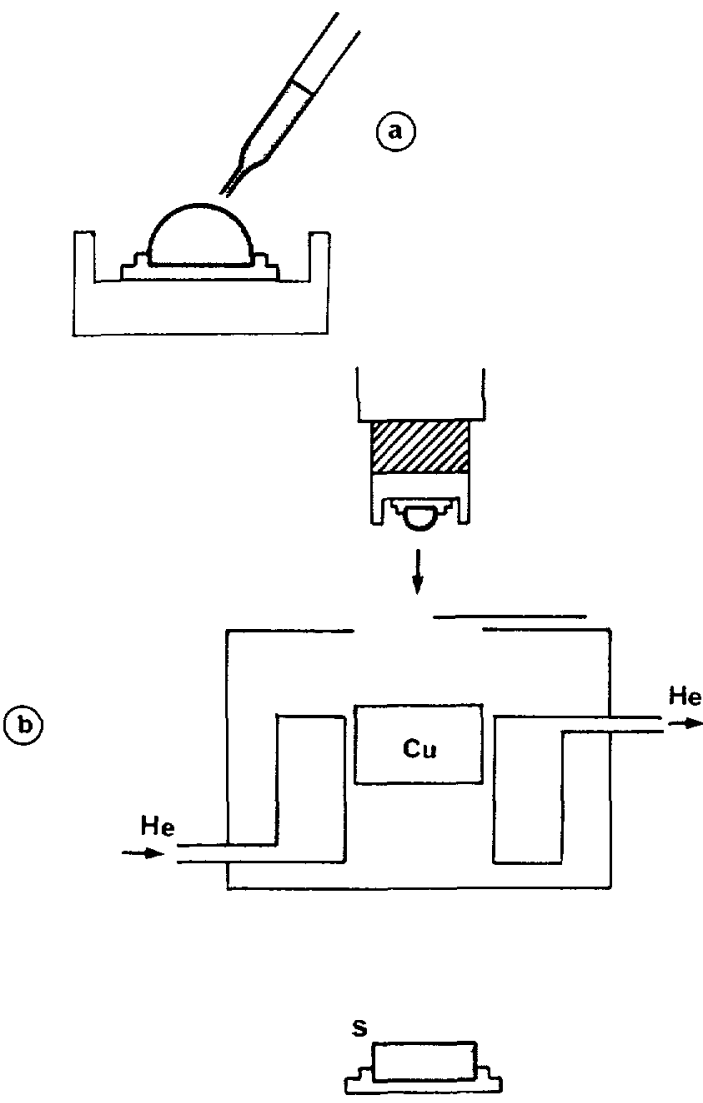

(c)

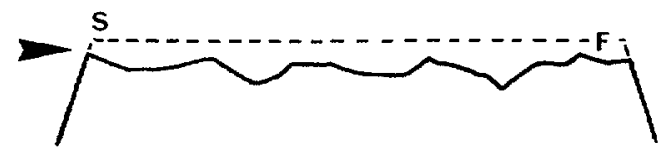

Fig. 1. - Cryofixation method. DNA cholesteric liquid crystalline samples are deposited onto copper discs that are sticked in a teflon cupel $1.1 \mathrm{~mm}$ deep (a). The sample is frozen by projection onto a copper block cooled down to liquid helium temperature (b), and the edge of the cupel serves as an abutment that avoids the complete squashing of the sample. During the fracture, the razor blade is oriented parallel to the flattened surface $S$ of the sample. The fracture surface $F$ is therefore on average parallel to this latter though it may present local reliefs (c).

or oblique relative to $C$, leading respectively to parallel alignments of DNA filaments, alternations of filaments parallel, oblique and normal to the fracture plane with a periodicity corresponding to half the helical pitch, $P_{\text {chol }} / 2$ (plate Ia) and periodic rows of nested arches (plate $\mathrm{Ib}$ ). Hence, freeze-fracture of a sample displaying no preferential orientation of the cholesteric layers is expected to generate mainly rows of nested arches with strong width variations that would correspond to the different slopes of oblique fracture planes relative to the cholesteric axis $C$. Conversely, archless cholesteric layers characterizing fracture planes perfectly parallel to the cholesteric axis would be less frequent and unidirectional molecular alignments highly unlikely. 

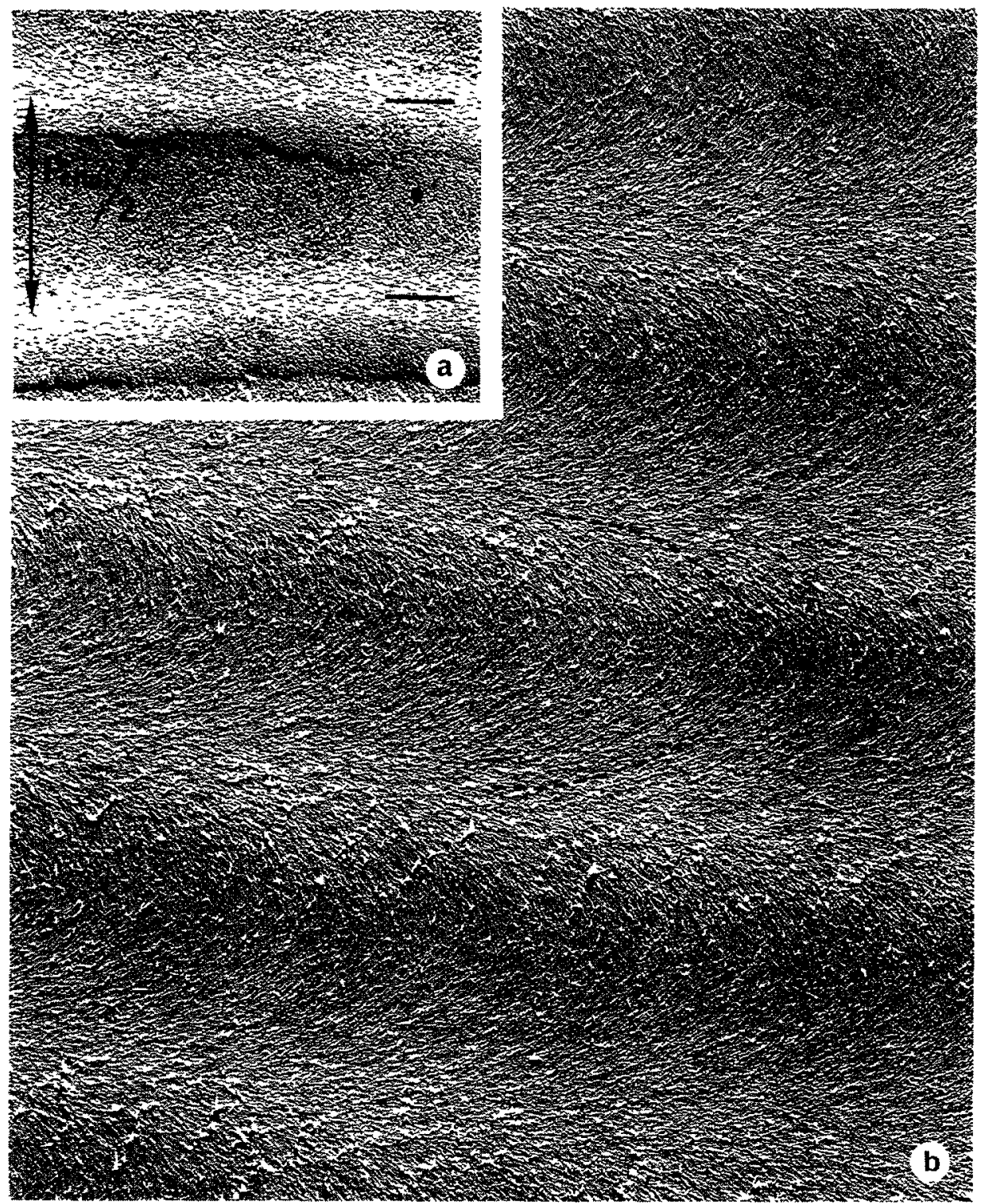

Plate I. - Freeze-fracture electron microscopy of DNA cholesteric liquid crystalline phase. (a) Archless cholesteric stratification observed on fracture planes parallel to the cholesteric axis. The periodicity corresponds to $P_{\text {chol }} / 2$. Lines and points respectively underline molecules parallel and normal to the observation plane (x 12600). (b) Rows of nested arches (dotted lines) drawn by the projection of the molecular orientations onto oblique fracture planes (x 13300).

Yet, in our experiments, alternating lines and point patterns (plate Ia) do occur as often as rows of nested arches (plate $\mathrm{Ib}$ ). Besides, the arches themselves are in most cases not drastically wider than $P_{\text {chol }} / 2$ : the fracture plane is therefore slightly oblique with respect to the cholesteric axis $C$. We hence put forward the hypothesis of a preferential orientation of the cholesteric axis that would tend to be parallel to the air interface. The observation of a free surface droplet, that shows that cholesteric layers do preferentially align normally to the air 
interface, strengthens this hypothesis. As expected, parallel alignments of molecules (not illustrated) are extremely rare, for, in any case, these patterns correspond to the less likely fracture planes.

3.2 DisTORTED CHOLESTERIC STRUCTURE. - Besides these three standard types of patterns, all the replicas frequently show multiple extra kinds of patterns that superimpose on the cholesteric stratification. The obtention of these distorted patterns is highly reproducible. They occur throughout the whole replica, even though regular cholesteric areas are locally observed (plate I). However, observation of the replicas does not allow us to estimate precisely the proportion of distorted to undistorted cholesteric domains within a given area. This point will be discussed further.

Besides, the frequency as well as the importance of the distortions are modulated with the polymer concentration in the phase. Indeed, the deformation of the cholesteric structure is stronger within the less concentrated areas, since alongside the isotropic phase, the cholesteric organization is in most cases entirely distorted. Figure 2 sketches the relationship between the occurrence or the distorted patterns and the DNA concentration in the cholesteric phase within a standard sample displaying a sizeable concentration gradient between its centre and periphery.

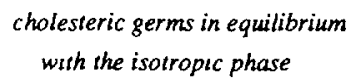

with the isotropic phase columnar hexagonal germs in equalibrium with the cholesteric phase

DNA concentration

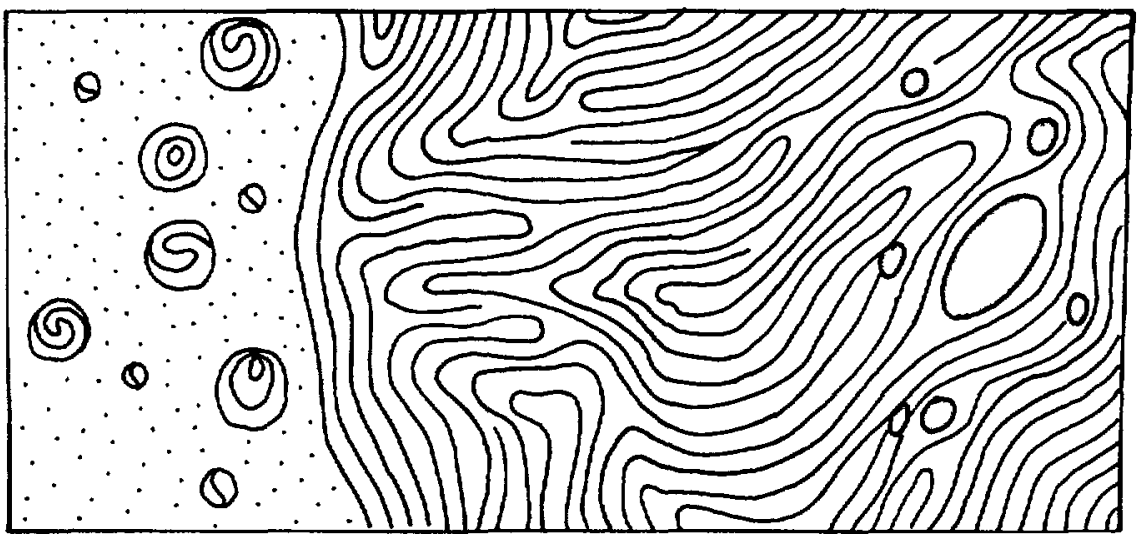

frequency of the

waved patterns

$+$

$+++$

$+$

$+$

Fig. 2. - Frequency of the waved patterns on replicas of samples showing strong polymer concentration gradient.

The distorted cholesteric patterns observed on the replicas can be classsified into three main types :

3.2.1. Periodic waves. - Periodic series of waves that are generated by molecules lying parallel or close to the fracture plane superimpose on the cholesteric stratification (plates II and III (arrows)). These waves are periodic along two orthogonal directions, on the one hand along the cholesteric layers and on the other hand along the cholesteric axis itself. Towards the first direction, their apparent periodicity $p_{\text {und }}^{\prime}$, that is measured on the micrographs, varies from 0.6 


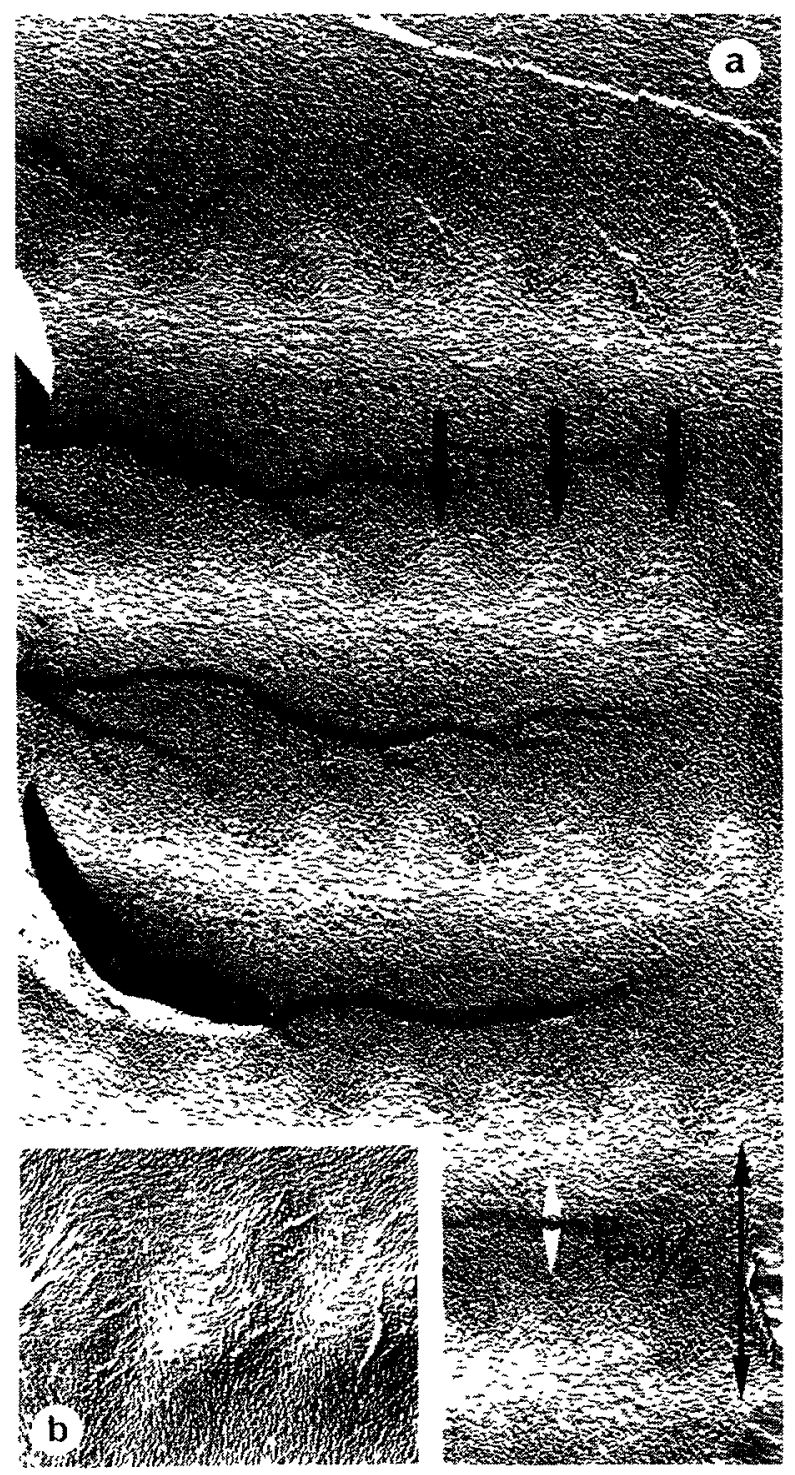

Plate II. - Periodic waves normal to the cholesteric stratification. Molecules parallel or close to the fracture plane draw small periodic waves on one side of each layer (arrows). Note that half of the layer appears undistorted (white arrowheads). The fracture plane is normal to the cholesteric stratification (a, $x$ 20500). Note in (b) the crenelled aspect of these waves, easily visible at higher magnification (x 21750).

to more than $2.5 \mu \mathrm{m}$. Note that, since fractures planes parallel to the cholesteric axis are not necessarily parallel to the wave direction, we do not have a direct access to the periodicity $p_{\text {und }}$ of these waves in space. It is hence only possible to settle that $p_{\text {und }}$ is smaller than the fifth of the cholesteric pitch $P_{\text {chol }}$, that varies in our samples from about 2.2 to $3.4 \mu \mathrm{m}$. Conversely, their amplitude can be directly measured as about $0.4 \mu \mathrm{m}$, on micrographs corresponding to fracture planes parallel to the cholesteric axis (plate IIa). These data are summarized in table I. Towards the second direction, the periodicity of the waves superimposes on the cholesteric one, since series of waves are separated by half the helical pitch $P_{\text {chol }} / 2$. 


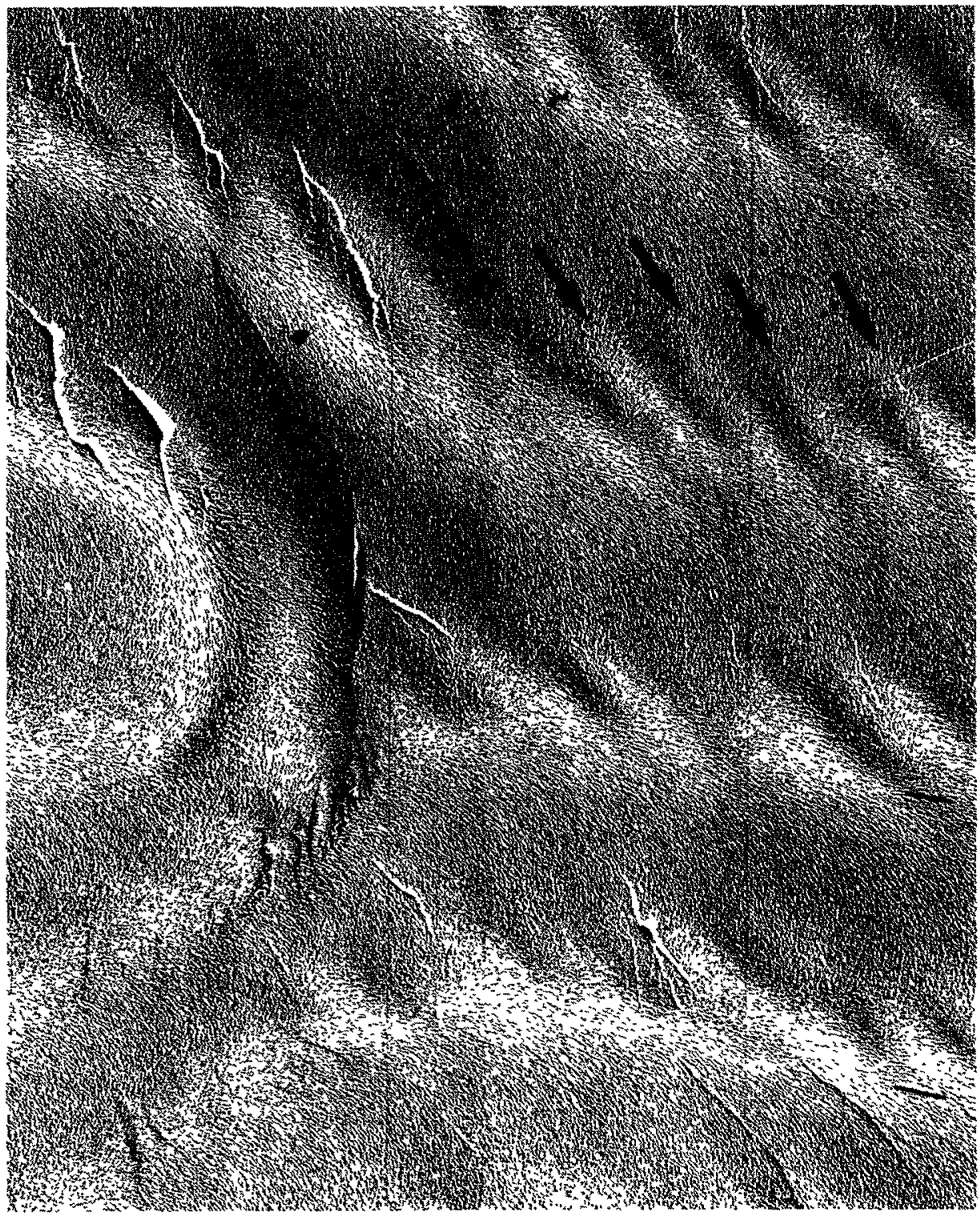

Plate III. - Periodic waves oblique to the cholesteric stratification. The fracture plane is oblique relative to the cholesteric layers. Half of the arches is disturbed by the occurrence of waves (arrows) drawn on one side of each layer by the molecules close to the fracture plane (indicated by lines). Note in the left part of the micrograph, that a disappearance of these waves is related to a change of the layer orientation due to an edge dislocation ( $x$ 13400).

The precise geometrical analysis of these two dimensional patterns allows the understanding of the main geometrical features of the three dimensional structure itself. Let us first recall that each cholesteric layer corresponds in space to a $180^{\circ}$ rotation of the molecular orientations along the cholesteric axis $C$. Obviously, the deformations of the molecular paths appear more clearly on the replicas as molecules lie parallel or close to the fracture plane, while they are hardly visible as molecules are close to the converse perpendicular situation. Within each cholesteric layer, the successive molecular levels shall therefore not appear equally distorted. 
Table I. - Period and amplitude of periodic waves measured on micrographs showing fracture planes normal to the cholesteric stratification.

\begin{tabular}{|l|c|}
\hline Cholesteric pitch Pchol & 2.2 to $3.4 \mu \mathrm{m}$ \\
\hline Measured undulation periodicity p'und & 0.6 to $2.5 \mu \mathrm{m}$ \\
\hline Undulation amplitude A & 0.37 to $0.44 \mu \mathrm{m}$ \\
\hline
\end{tabular}

On plates II and III, molecules corresponding to similar obliquities relative to the fracture plane, for example on both sides of the band drawn by those lying parallel to the fracture plane, do not display the same deformations (the waves are drawn asymmetrically within each cholesteric layer). The intensity of the deformation of the cholesteric structure is therefore not monotonous but modulated by the variations of the molecular orientations along the cholesteric axis. This modulation, combined with a two-dimensional visualization of the structure, raises apparent regular semi-layers on the replicas (plate II, arrowheads), the other half of the cholesteric layer being strongly distorted by a series of waves.

On plate IIa, where the periodic stratification devoid of arches corresponds to a fracture plane parallel to the cholesteric axis, the waves form normally to the cholesteric layers, their axis being parallel to the cholesteric one. Conversely, on oblique fracture planes that are characterized by the presence of arches, the waves appear oblique to the cholesteric layers (plate III, arrows). Their axis is oriented inwards the parabolic arches drawn by the molecular orientations in projection onto the fracture plane ; the part of the cholesteric layer that is mostly distorted corresponds, in the regular arches of reference, to the molecular orientations that lie normal to the direction of the waves. In both cases, the axis of the waves is therefore normal to the molecular orientations of the initial cholesteric structure. These two kinds of waved patterns (plates II and III) correspond to different views of the same three-dimensional phenomenon and reveal the occurrence of periodic undulations of the molecular layers towards the direction of the cholesteric axis.

3.2.2 Undulated arches. - Some areas of the replicas do likewise present slight undulations of the arches themselves (plate IVa). We measured on several micrographs the wavelength of this phenomenon as 0.5 to $0.6 \mu \mathrm{m}$. These patterns are rather uncommon and only encountered in the vicinity of the isotropic phase, i.e. in areas that correspond to a smaller polymer concentration of the cholesteric phase.

3.2.3 Strongly distorted patterns. - Last, at the immediate vicinity of the undulated arches, strongly distorted patterns are observed (plate IVb). The cholesteric periodicity is hardly recognizable, as molecular orientations undulate in all directions. This situation occurs frequently close to the isotropic border.

\section{Models.}

Let us recall the usual representation of the cholesteric liquid crystalline organization. It is schematized by a succession of equidistant planes that do not exist in reality but simplify the drawing. The average molecular directions are parallel within a given plane and rotate of a constant angle from one plane to another (Fig. 3a), the structure being left-handed in DNA 

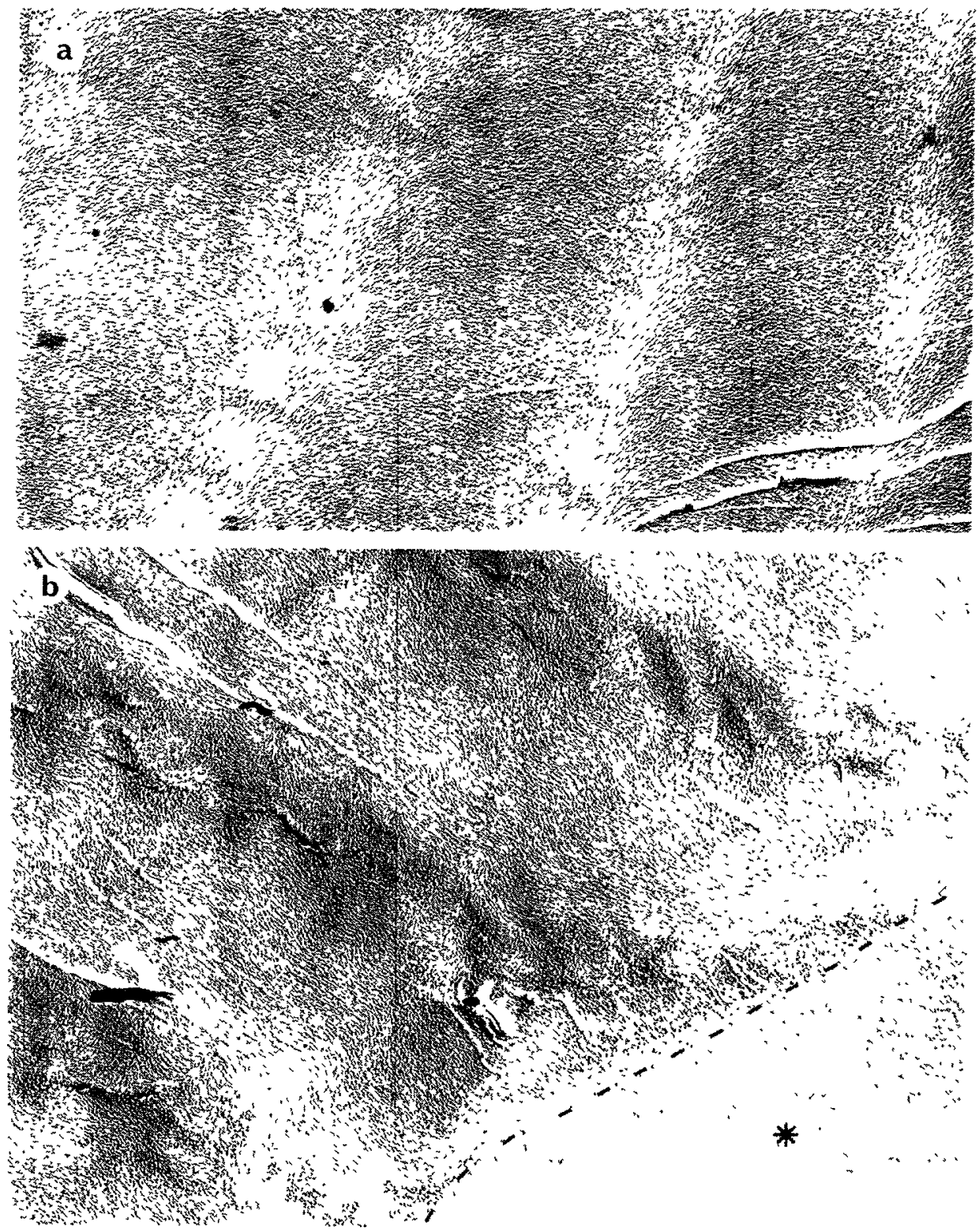

Plate IV. - (a) Undulated arches (x 14000). (b) Strongly distorted features showing molecular undulation within all directions observed at the isotropic border (dotted lines). The periodicity of the structure is hardly recognizable. The star indicates the isotropic phase (x 11100).

cholesteric mesophases [15]. Note that the continuous twist is represented by discrete planes in this figure. In spite of the fact that these planes cannot be considered as physical entities (except in mesophases formed by long chain polymers where such a layering often arises), we shall take them into account to model distortions of the cholesteric structure.

Let us first consider the wave phenomenon. Since the axis of the waves is always normal to the molecular orientations of the initial cholesteric structure, we can deduce that, in space, these patterns correspond to periodic undulations of the molecular layers. These data are taken 

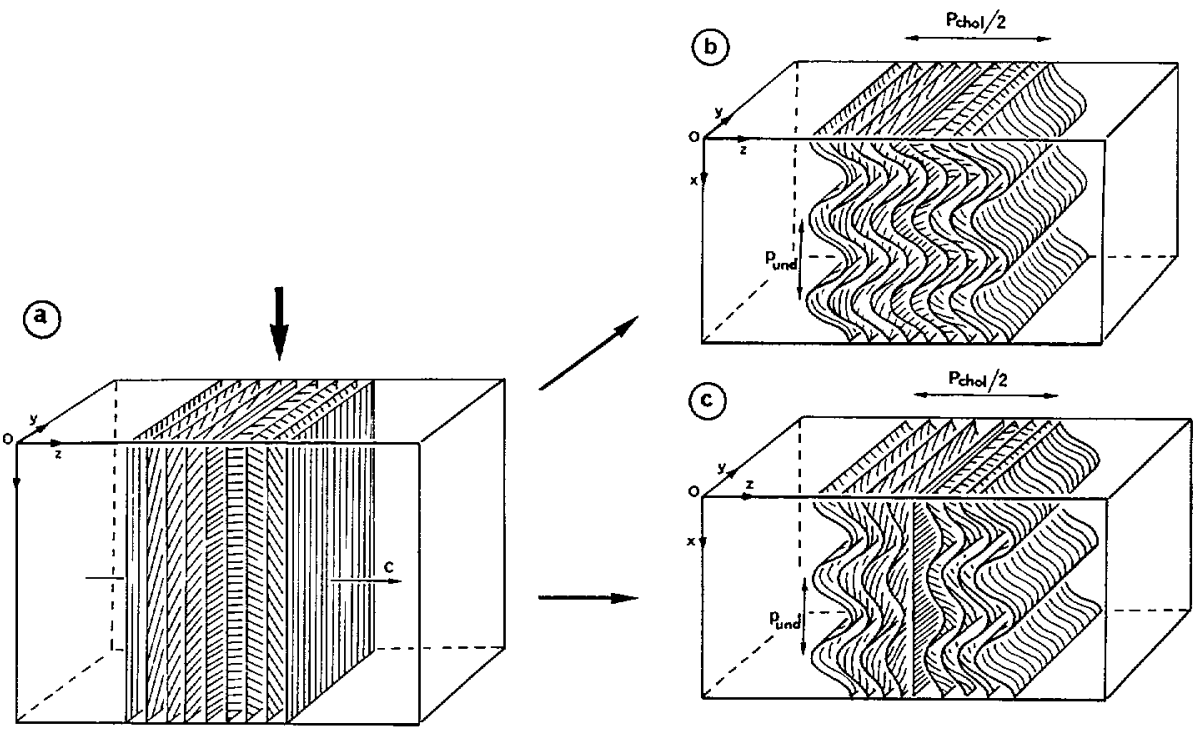

Model $A_{1}$

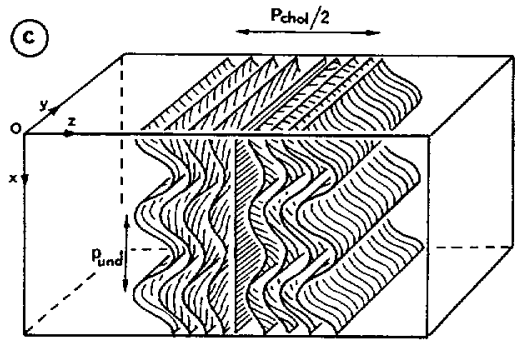

Model $A_{2}$
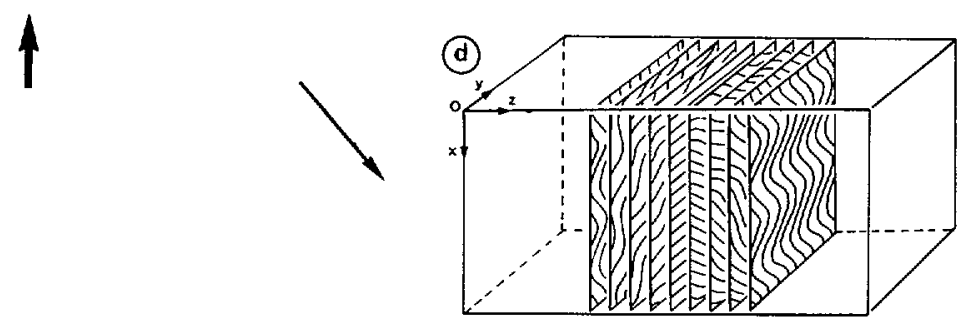

Model B

Fig. 3. - Different models of a cholesteric sample subjected to a compressive stress applied normally to the stratification (arrows). Let a Cartesian coordinate system $0 x y z$ be associated with a cholesteric slab, with $x 0 y$ normal to the cholesteric axis $C$. The $y 0 z$ plane corresponds to the surface of the sample. Model $A_{1}$. The fictitious planes are distorted into sinusoidal surfaces in the compressive direction $0 x$ (b). Model $\mathrm{A}_{2}$. The amplitude of the sinusoidal distortion of the cholesteric planes is modulated with the periodic variation of the molecular orientations relative to the stress direction $0 x(\mathrm{c})$. Model $\mathrm{B}$. There is no plane deformation : the molecular paths transform into sinusoidal plane curves which amplitude can also be modulated according to the initial molecular orientations (d).

into account to propose a basic model of these deformations as the sinusoidal undulation of the cholesteric fictitious planes (Fig. $3 b$, model $A_{1}$ ).

Besides, since the periodicity of the undulation of the molecular layers superimposes on the cholesteric stratification, it is possible to assume that the planes are not equally affected by the compressive stress. Indeed, the molecular orientations relative to the flattening direction (indicated by arrows in Fig. 3) vary and there is no reason for the monotony of the response of the structure to the stress. We therefore modulate the amplitude of the sinusoidal deformations in model $\mathrm{A}_{2}$ (Fig. 3c). This modulation obviously leads to a structure that periodically presents a nearly undistorted area surrounding the molecules perpendicular to the compressive force direction.

Even though experimental data suggest that the deformation encountered within our samples may be close to that sketched as model $A_{2}$ in figure $3 c$, we shall first focus our attention on model $A_{1}$ (Fig. $3 b$ ), on the one hand for the sake of simplicity in order to settle the main geometrical properties of such structures, and on the other hand for the biological relevance of this model that will further be pointed out. 
Finally, in order to explain the patterns of plate IV, we consider additional modifications of molecular orientations that correspond to the sinusoidal undulation of the molecular orientations within their planes (Fig. 3d, model B). Moreover, we shall consider a combination of both models, corresponding to the sinusoidal undulations of the molecular paths upon sinusoidal surfaces.

4.1 MOdel $A_{1}$. Sinusoidal undulation of the CHOlesteric layers (Fig. 3b). - We consider the theoretical situation of a cholesteric slab submitted to a compressive stress applied normally to the cholesteric axis. Hence, the cholesteric pseudoplanes transform into parallel sinusoidal surfaces undulating in the flattening direction (associated with the $0 x$ axis in Fig. 3b). We arbitrarily set the undulation amplitude $A$ and wavelength $p_{\text {und }}$ in the range of experimental data (Tab. I) as $A=P_{\text {chol }} / 8$ and $p_{\text {und }}=P_{\text {chol }} / 4$; note however that the wavelength $p_{\text {und }}$ is certainly smaller, but this arbitrary choice simplifies the drawing.

4.1.1 Intermolecular distances variations. - Let us first recall that the periodic undulation of a lamellar structure (Fig. 4a) can be achieved either by the formation of curvature walls keeping the thickness of the layers constant (Fig. 4b), or by allowing variations of the thickness of the layers that permit true sinusoidal surfaces to pile up (Fig. 4c). In the case of smectic liquid crystals, the latter possibility is ruled out as the layer thickness cannot vary (if one excludes the occurrence of molecular tilt within the layers), when in cholesteric liquid crystals local pitch variations can be taken into account. The long range organization of cholesteric liquid crystals can be considered as lamellar, though it is rather similar to nematics on the molecular scale.

Let $d_{0}$ be the initial interplanar spacing (Fig. 4a). We introduce a sinusoisal deformation of the planes in the $0 x$ direction in order to obtain a set of nested parallel sinusoidal surfaces (Fig. 4c). The intersurface spacing $d$ then presents periodic local variations in the $0 x$ direction :

$$
d=d_{0}|\cos [\arctan (a k \cos (k x))]|
$$

where $a=A / 2$ and $k=2 \pi / p_{\text {und }}$.

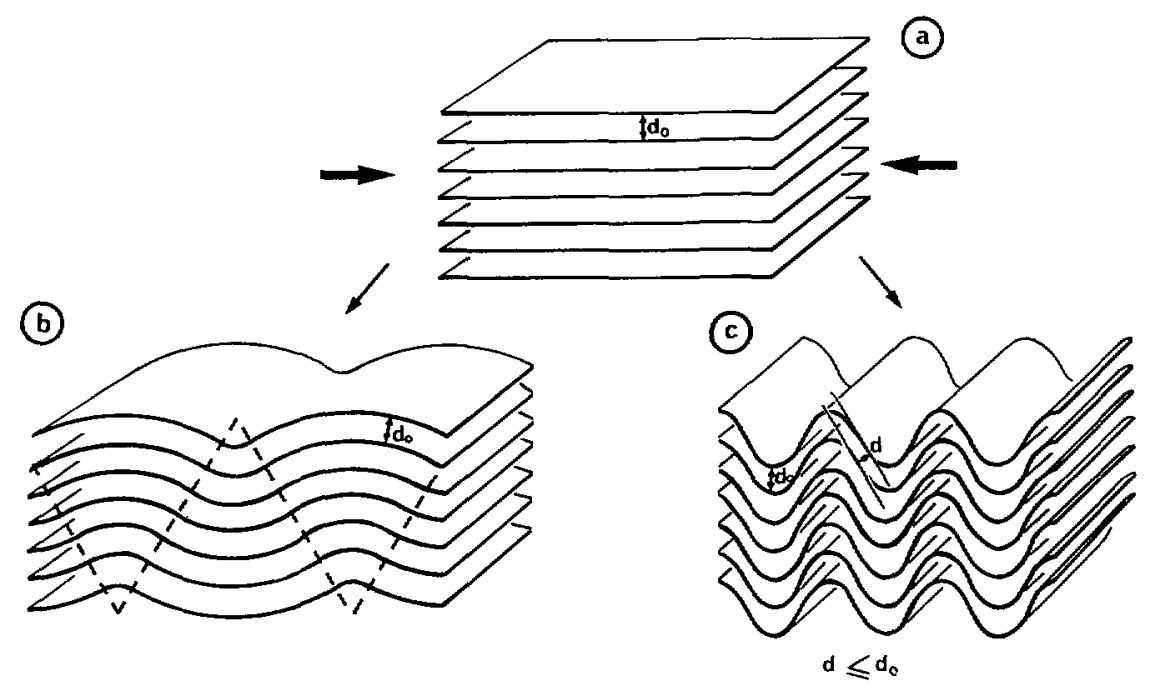

Fig. 4. - Let a lamellar system with an interplane distance $d_{0}$ be (a). In $\mathbf{b}$, the thickness of the layers $d_{0}$ is kept constant. There is formation of curvature walls (dotted lines). In c, unconstancies of the thickness of the layers are introduced : parallel sinusoidal surfaces can pile up. The distorted structure is periodic and can be deduced by translation. 
Local interhelix spacing, proportional to $d$, is therefore no longer constant but varies periodically within the structure. The minimal intermolecular distance value, encountered at the inflexion lines, will depend on the $A / p_{\text {und }}$ ratio. Note that in the model sketched in figure $3 \mathrm{~b}, d \leqslant d_{0}$ with $d=d_{0}$ at the hills and valleys of the sinusoidal distortion. Molecular spreading out at the hills and valleys $\left(d>d_{0}\right)$ alternating continuously with the highest stacking at the inflexion $\left(d<d_{0}\right)$ could have also been taken into account.

4.1.2 Molecular paths. - The line integrals associated with the director field are successively parallel, oblique and normal to the flattening direction $0 x$. After compression, lines parallel to the undulation direction $0 x$ will transform into sinusoids (Fig. 5a), when those normal to $0 x$ will remain straight (Fig. 5b). The in-between situation is somewhat less obvious. Let us consider the three perpendicular axes $0 x y z$, the $y 0 z$ plane corresponds to the sample surface and the $0 x$ axis to the flattening direction. We distort the plane $x 0 y$ into the sinusoidal surface $z=a \sin (k x)$. Considering any line $D_{0}$ of the $x 0 y$ plane, let $\alpha$ be the angle of $D_{0}$ to $0 x$. The line $D_{0}$ described by

$$
\begin{aligned}
& x=y / \tan \alpha \\
& z=0
\end{aligned}
$$

transforms into the somewhat complex curve $S$ (Fig. 5c):

$$
\begin{aligned}
& x=k^{-1} E^{-1}\left[a / \sqrt{\left(a^{2}+k^{-2}\right)}, y /\left(\tan \alpha \sqrt{\left(a^{2}+k^{-2}\right)}\right)\right] \\
& z=a \sin \left(E^{-1}\left[a / \sqrt{\left(a^{2}+k^{-2}\right)}, y /\left(\tan \alpha \sqrt{\left(a^{2}+k^{-2}\right)}\right)\right]\right)
\end{aligned}
$$

with $E(K, \Phi)=\int_{0}^{\Phi} \sqrt{\left(1-K^{2} \sin ^{2} \phi\right)} \mathrm{d} \phi$ (see appendix).

Letting $\alpha$ vary from 0 to $\pi / 2$, we obtain a family of periodic curves inscribed inside elliptic cylinders, that transform continuously into sinusoids at $\alpha=0$ and discontinuously into lines at $\alpha=\pi / 2$ (Fig. 6b). They project as eight-shaped curves onto transverse sections of the cylinders as sketched in figure 6a. Their ellipticity is a continuous function of $\alpha$, except at $\alpha=\pi / 2+n \pi$ ( $n$ integer) where a discontinuity arises in the structure.
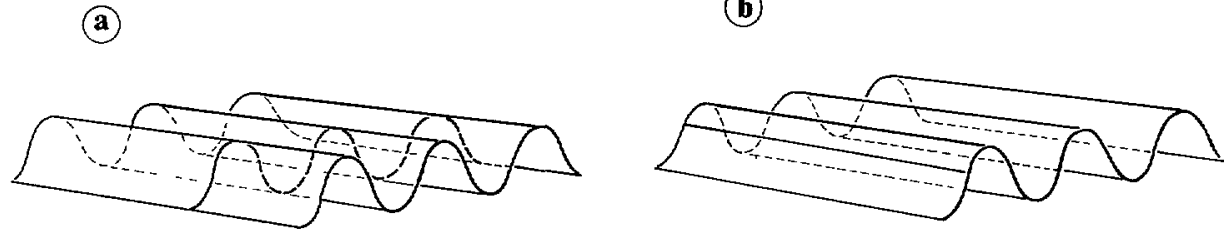

(c)

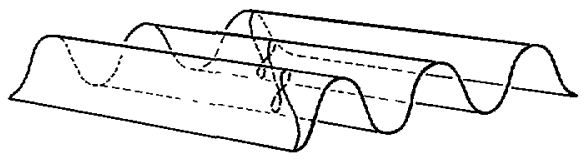

Fig. 5. - Molecular paths within the distorted structure. Three situations have to be taken into account : sinusoidal curves (a), lines (b) and complex periodic space curves (c). They correspond to the three different molecular orientations in the initial structure relative to the compression direction, i.e. parallel. normal and oblique, respectively. 
$\alpha$

(a)

(a) $\begin{array}{lll}\alpha=0 & \alpha=\frac{\pi}{8} & \alpha=\frac{\pi}{4}\end{array} \quad \infty=\frac{3 \pi}{8} \quad \alpha=\frac{\pi}{2}$

(23)
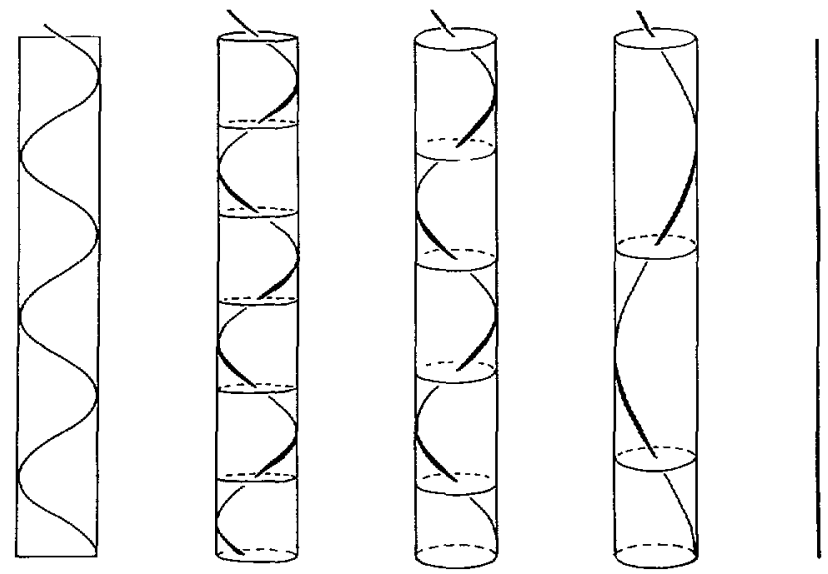

$\alpha$

(b)

$\begin{array}{llccc}a=0 & a=\frac{\pi}{8} & \alpha=\frac{\pi}{4} & \alpha=\frac{3 \pi}{8} & a=\frac{\pi}{2} \\ & \infty & \varnothing & \end{array}$

(b2)
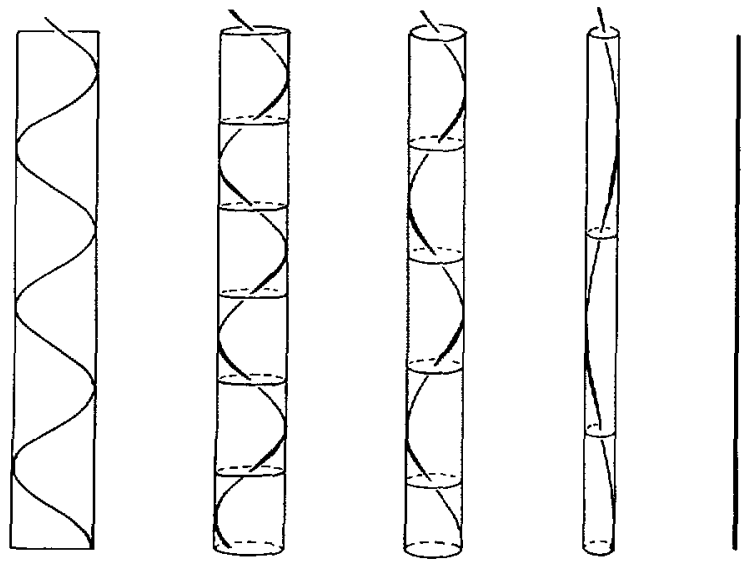

Fig. 6. - (a) Model $A_{1}$. In space, molecular paths within the distorted structure can be described as a family of periodic complex space curves inscribed inside elliptic cylinders whose ellipticity is a function of $\alpha$, the angle of the initial molecular orientation to the $0 x$ axis (flattening direction) $\left(a_{2}\right)$. The projection of molecular paths onto the transverse sections of the cylinders eases their understanding $\left(a_{1}\right):$ they draw eight-shaped curves that flatten continuously into a segment at $\alpha=0$ and transform abruptly into a point at $\alpha=\pi / 2$. (b) Model $A_{2}$. Molecular paths can be described as a family of periodic complex space curves inscribed inside elliptic cylinders that are function of $\alpha\left(b_{2}\right)$. The projection of these curves onto the transverse section of the cylinders draws eight-shaped curves that flatten into a segment at $\alpha=0$ as in model $A_{1}$ and reduce continuously to a point as $\alpha$ tends towards $\pi / 2\left(b_{1}\right)$. 


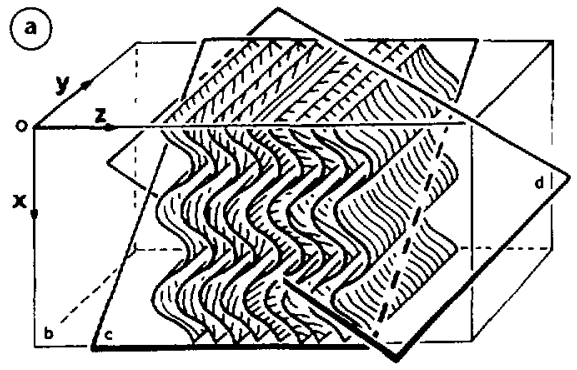

(b)

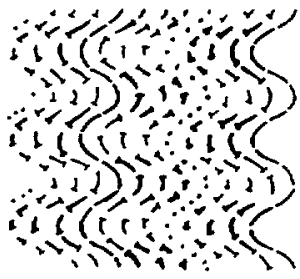

(c)

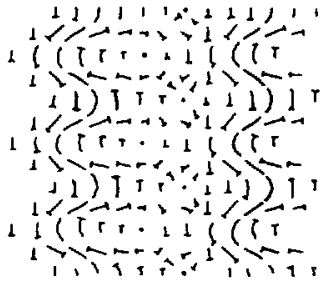

(d)

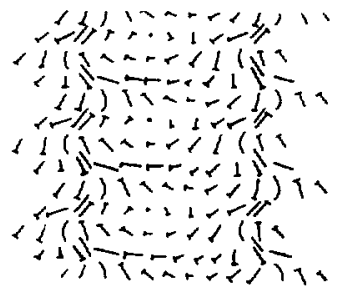

Fig. 7. - Model $A_{1}$. Theoretical patterns obtained by the projection of the molecular orientations onto different section planes using the nail drawing convention : (b) section plane parallel to $x 0 z$ (frontview) : double twist of opposite handedness areas appear in grey, when those of double twist of same handedness areas are left white. These double twist areas are separated by single twist surfaces that can be either planes or sinusoidal surfaces. (c) section plane parallel to $0 z$ and oblique to $0 x$ and $0 y$. (d) section plane oblique to the three axes.

We must now recall that the molecules we are dealing with cannot be idealized as infinite chains since their length, i.e. $50 \mathrm{~nm}$ is about ten times smaller than the undulation periodicity $p_{\text {und }}$. As the above mentioned complex curves introduce within the structure strong twist variations that will not be set out here, we can imagine that the average director $\mathbf{n}$ does not exactly follow these curves: $\mathbf{n}$ may somehow be let loose to espouse the sinusoidal curve drawn by the intersection of the sinusoidal surface and the plane $y=x \tan \alpha$, thus reducing twist variations. 


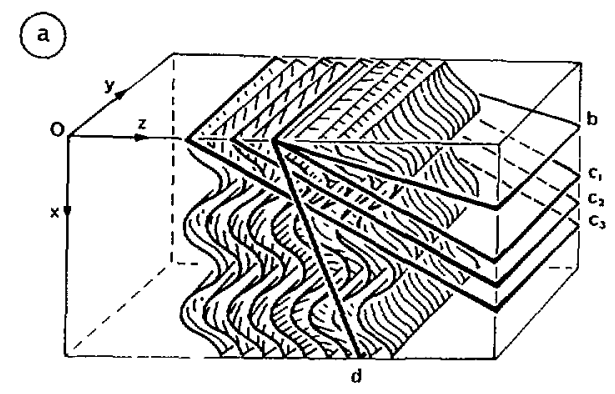

(b)

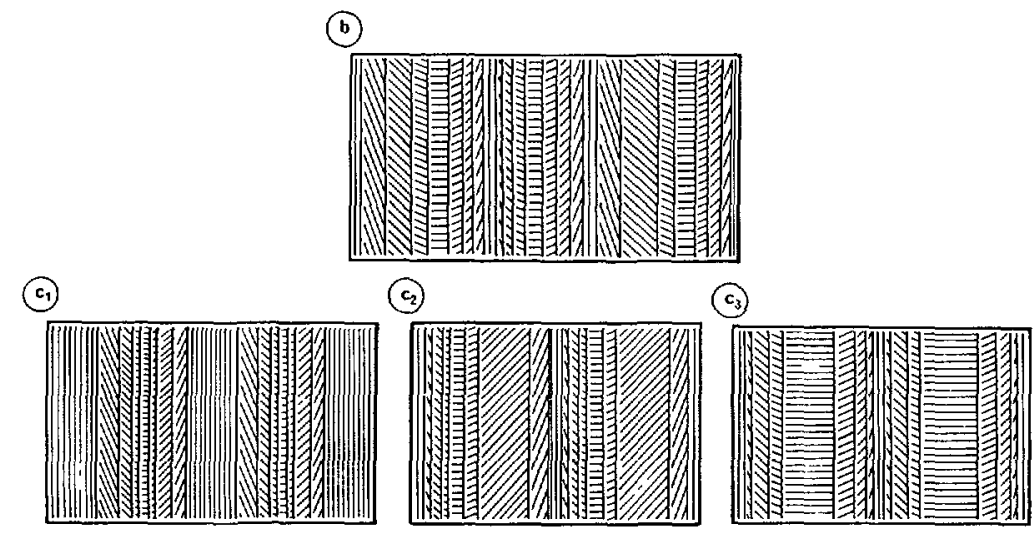

(d)

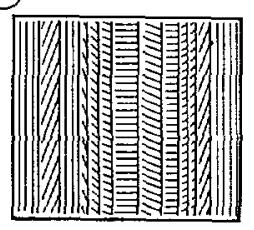

Fig. 8. - Model $A_{1}$. Theoretical section planes parallel to $0 y$. These section planes generate periodic or quasi periodic rows of nested arches according to $\beta$, the angle of the fracture plane to the surface plane y0z. (b) Quasiperiodic rows of dissymmetric nested arches. (c) Peculiar features of $P_{\text {chol }} / 2$ periodicity. The arches can be separated by wide unidirectional alignments of fibrils $\left(c_{1}\right)$, dissymmetric $\left(c_{2}\right)$, or flattened $\left(c_{3}\right)$. (d) General feature generated when $\beta>\pi / 2-\arctan (a k)$. The arches are no longer parabolic but present sigmoidal segments.

4.1.3 Theoretical sections of the model. Geometrical analysis. - Multiple theoretical sections of the model were established according to different orientations (Figs. 7, 8). We deal here with a periodic 3D structure that is continuous and periodic in two directions. It is associated with a Cartesian coordinate system $0 x y z$ with $0 z$ as the direction of cholesteric periodicity and $0 x$ as that of the sinusoidal distortion. The structure is constant in the $0 y$ direction. Let us consider any arbitrary discrete representation of this system as a lattice of equiphase lines parallel to $0 y$ (Fig. 9). A section plane that would not encounter any of these lines except the origin therefore generates quasi periodic patterns, when any other section plane generates periodic patterns. This topic has been pointed out and discussed by Gaill and Mosseri [16]. 


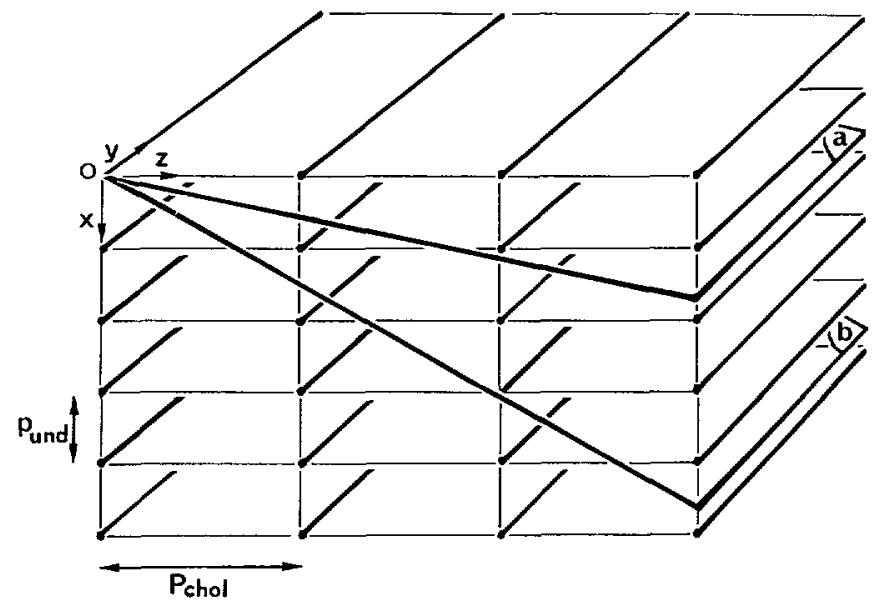

Fig. 9. - Discrete schematization of models $A_{1}$ and $A_{2}$. Equiphase lines are parallel to $0 y$ and form a lattice that is periodic in the $0 x$ and $0 y$ directions. Section planes parallel to $0 y$ that do not encounter more than one of these lines therefore generate quasi periodic patterns (a). Periodic patterns are obtained on section planes periodically equiphase lines (b).

The $y 0 z$ plane is parallel to the sample surface. Fracture planes normal to $y 0 z$ are therefore very unlikely and will not be taken into account here. We then consider three main families of fracture planes that may lie parallel to $0 y$, parallel to $0 z$ or oblique to the three axes. The last two theoretical situations are schematized in figures $7 \mathrm{c}$, d. These families of fracture planes always generate periodic patterns for they intersect any line of figure 9 lattice. Molecular orientations projected onto a section plane are conventionally schematized by lines, nails and points when molecules lie parallel, oblique and normal to the section plane. This representation facilitates the visualization of local twist and splay phenomena. We shall here mainly dwell on twist variations within the distorted structure.

Let us first recall the three fundamental arrangements within fibrous systems, splay, twist and bend as they have been locally defined by Frank [17]. Let $\mathbf{n}$ be the unit vector associated with the preferred molecular orientation at any point. $\mathbf{n}$ is associated with a local system of perpendicular axes $0 x_{n} y_{n} z_{n}$, $\mathbf{n}$ being parallel to $0 z_{n}$ (see Fig. 10a, redrawn after Frank [17]). The two components of splay, twist and bend are respectively defined by:

$$
\begin{array}{ll}
s_{1}=\partial n_{x_{n}} / \partial x_{n} & s_{2}=\partial n_{y_{n}} / \partial y_{n} \\
t_{1}=-\partial n_{y_{n}} / \partial x_{n} & t_{2}=\partial n_{x_{n}} / \partial y_{n} \\
b_{1}=\partial n_{x_{n}} / \partial z_{n} & b_{2}=\partial n_{y_{n}} / \partial z_{n} .
\end{array}
$$

Regular planar cholesteric structure corresponds to a pure single twist situation : all the components, with the exception of $t_{1}$, are nil, with $0 x_{n}$ chosen as the cholesteric axis and thus corresponding to the maximal twist direction [18].

The sinusoidal distortion of the planes obviously leads to the emergence of double twist and bend phenomena accompanied by splay deformations allowed by the small molecular length relative to the amplitude and wavelength of the distortion (besides, the DNA density in the distorted structure is not constant as shown above).

Local double twist is more clearly visualized when $\mathbf{n}$ is normal to the section plane. Let us for example consider and zoom molecules that lie normal to the section plane in figure $7 \mathrm{c}$. We 
(a)
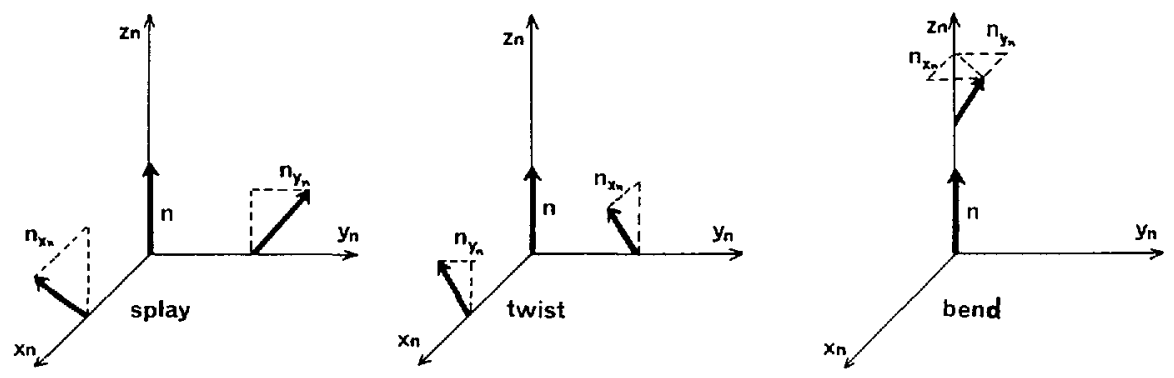

(b)
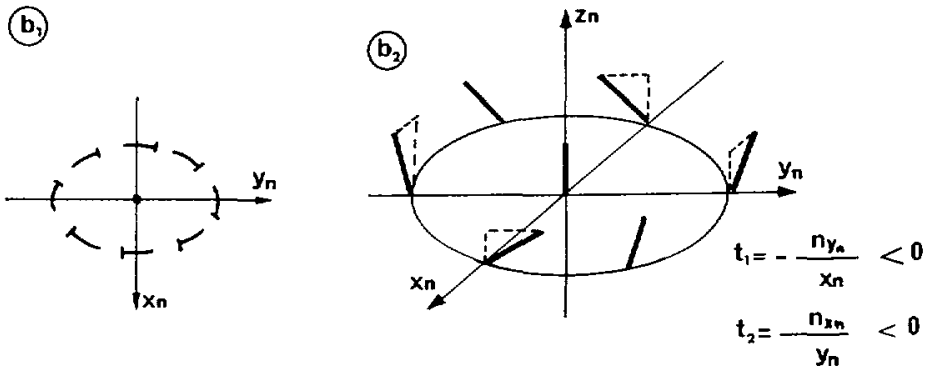

(c)
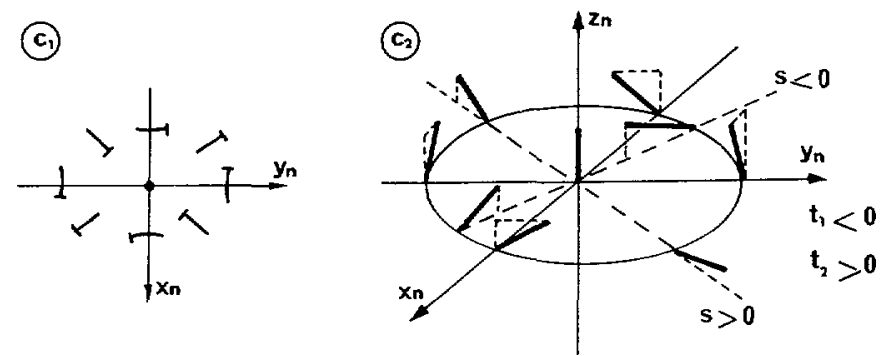

Fig. 10. - Let $0 x_{n} y_{n} z_{n}$ be a local coordinate system associated with the unit vector corresponding to the molecular orientation $n$ at any point. The curvature components splay, twist and bend are redrawn after Frank [18] (a). Double twist situations encountered in figure $8 \mathrm{c}$ are considered more precisely. We distinguish double twist of same handedness (left) (b) and double twist of opposite handedness (c). Both situations are schematized using Frank's convention $\left(b_{2}, c_{2}\right)$. Note that for the sake of simplicity, we schematized here pure twist situations when we deal in reality with twist and splay combination, at the exception of the hills and valleys where the splay is nil.

encounter two different situations : $t_{1}$ and $t_{2}$ may both be negative, i.e. both left-handed (Fig. 10b), or $t_{1}$ may be negative when $t_{2}$ is positive, i.e. $t_{2}$ is right-handed (Fig. 10c). In the latter case, the continuous transition from a negative to a positive twist is achieved by a local intermediate pure splay situation.

Series of oblique section planes therefore permitted us to settle double twist phenomena at any point. The situation within the whole distorted structure is summarized in figure $7 \mathrm{~b}$. This cholesteric distorted structure thus consists of a periodic mosaic of domains in which the 
double twist is alternatively of same and opposite handedness. This mosaic has two periodicity directions. The sign of $t_{2}$ changes when $\alpha=0+n \pi / 2$, with $n$ integer, and at the inflexion lines of the sinusoidal surfaces.

A quantitative description of $t_{1}$ and $t_{2}$ at the hills and valleys of the sinusoidal surfaces is given in the appendix; it agrees with our nail analysis and besides shows that extreme values of $t_{2}$ are obtained for $\alpha=\pi / 4+n \pi / 2$, with $n$ integer.

Let us now consider the family of fracture planes parallel to $0 y$. This situation is recalled in figure 8 and produces arched patterns that slightly differ from regular ones. Note that these features do not require the use of nail drawing. As above recalled the structure is constant in the $0 y$ direction; these fracture planes are therefore parallel to the equiphase lines and thus the only ones that may possibly produce quasi periodic patterns. Let $\beta$ be the angle of the fracture plane to the sample surface, i.e. the $y 0 z$ plane (see Fig. 9). The rise of periodic and quasi periodic patterns is in this case related to the values of $\tan \beta$. Let us consider the general equation of the lattice lines:

$$
\begin{aligned}
& z=n P_{\text {chol }} \\
& x=m p_{\text {und }}
\end{aligned}
$$

with $n$ and $m$ integers. When $\tan \beta=n / m\left(P_{\text {chol }} / p_{\text {und }}\right)$, the fracture plane periodically encounters these lattice lines and therefore generates periodic patterns. If we consider $P_{\text {chol }} / p_{\text {und }}$ as an integer, periodic patterns will be obtained for any rational value of $\tan \beta$, whereas quasi periodic patterns correspond to irrational values of $\tan \beta$. Note that, even when $\tan \beta$ is rational, the periodicity of the arched patterns may not be visible, for the period may be much greater than the sample size (Fig. 8b) [16]. In figure $8 \mathrm{c}$ we schematized the very particular situation where the periodicity of the features corresponds to $P_{\text {chol }} / 2$. As the fracture plane successively passes through different DNA density areas, the arches drawn by the molecular orientations projected onto the fracture plane are separated by wide unidirectional alignments of fibrils (Fig. $8 \mathrm{c}_{1}$ ), dissymmetric (Fig. $8 \mathrm{c}_{2}$ ) or flattened (Fig. $8 \mathrm{c}_{3}$ ). In fact these fracture planes generate a wide range of arched patterns out of which we arbitrarily selected the examples of figure 8 . Lastly, if $\tan \beta>\pi / 2-\arctan (a k)$, the arches drawn on the section plane will no longer be parabolic but will present sigmoidal segments (Fig. 8d).

4.1.4 Comparison with the micrographs. - All these situations are encountered within the replicas, but we must mention that quasi periodic rows of nested arches were most of the time difficult to distinguish from periodic ones because of their pseudo regularity. Besides, the distortion of the cholesteric structure is not the only possible origin of the dissymmetry or flattening of the arches that may also be related to molecular displacements occurring during the fracturing process.

The two situations shown in figures $7 \mathrm{c}, \mathrm{d}$ are compared to the waved patterns of plate II and plate III in figures $11 \mathrm{a}, \mathrm{b}, \mathrm{c}_{1}, \mathrm{~d}_{1}$. Molecules lying parallel or close to the fracture plane draw periodic waved patterns, asymmetrically located within the layers. These waves can appear normal or oblique to the stratification; besides the latter situation displays semi-archlike features. Nonetheless, these features are not alike in the theoretical and observed situations : as already described, electron microscopy data show an undistorted area within each layer that does not exist in the theoretical patterns.

This model seems therefore to describe the kind of phenomenon occurring within the samples but the observed waved patterns slightly differ from the theoretical ones. We henceforth consider a variation of this model that will take into account modulations of the behaviour of the molecules with their relative orientation to the flattening direction. 
(a)

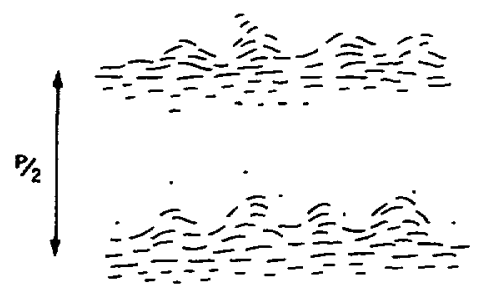

(b)

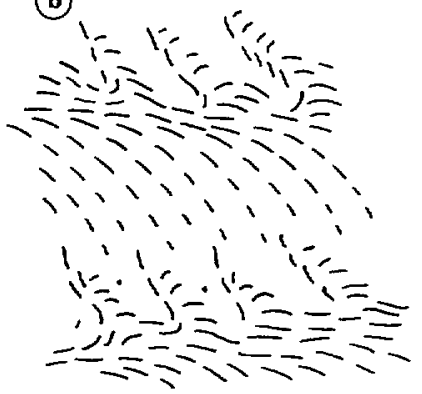

Experimental (c)

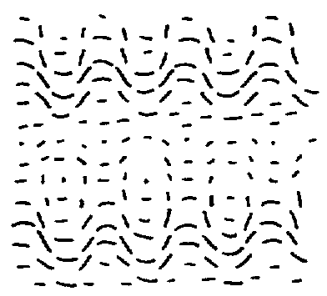

(d)

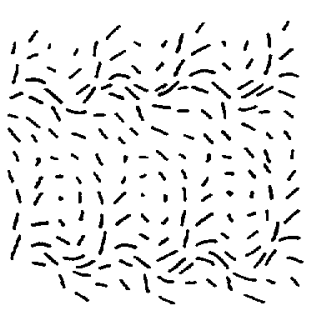

Model A1 (c)

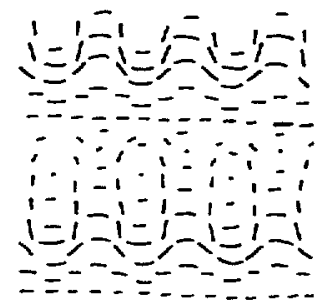

(d3)

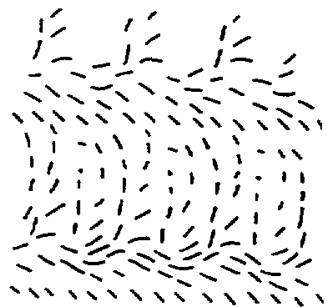

Model $\mathrm{A}_{2}$

Fig. 11. - Comparison of the observed patterns (a,b) with the theoretical sections of the models (c, d). a and $b$ are redrawn after plates II and III. $c_{1}$ and $d_{1}$ are redrawn after figures $6 c, d$ (model $A_{1}$ ). $c_{2}$ and $d_{2}$ are redrawn after figures $12 \mathrm{c}, \mathrm{d}\left(\operatorname{model} \mathrm{A}_{2}\right.$ ).

4.2 Model $\mathrm{A}_{2}$. Undulation of the ChOlesteric Layers With AMPLITUde MOdULATION (Fig. 3c). - Let us now resume our first model and introduce modulations of the deformation of the cholesteric planes according to the molecular orientation relative to the flattening direction. We allow the amplitude of the sinusoidal deformation to vary with the compressive force component along the molecular direction, i.e. as :

$$
A=A_{\max }|\cos \alpha|
$$

$\alpha$ being the angle of the molecular orientation to the flattening direction. We keep the wavelength and maximal amplitude values arbitrarily chosen for figure $3 \mathrm{~b}$, i.e. $p_{\text {und }}=P_{\text {chol }} / 4$ and $A_{\max }=P_{\text {chol }} / 8$ (Fig. $3 \mathrm{c}$ ).

4.2.1 Variations of the intermolecular distances. - The amplitude modulation creates a molecular stacking within the surfaces themselves. This stacking varies continuously with $\alpha\left({ }^{1}\right)$. After figure $3 \mathrm{c}$, this stacking is obviously maximal for $\alpha=\pi / 2+n \pi$ and nil for $\alpha=0+n \pi$, with $n$ integer.

(') Let $d_{1}$ be the interhelix spacing within a given surface, then we can settle:

$$
d_{1}(\alpha)=d_{1_{0}} \int_{0}^{\pi} \sqrt{\left(1+\left(a_{\max } k \cos \alpha\right)^{2} \cos ^{2}(k x)\right)} \mathrm{d} x / \int_{0}^{\pi} \sqrt{\left(1+a_{\max }^{2} k^{2} \cos ^{2}(k x)\right)} \mathrm{d} x
$$

with $a=A / 2$ and $k=2 \pi / p_{\text {und }}$ and $d_{\mathrm{l}_{0}}$ being the initial interhelix spacing. 
Besides, and as in our first model, interhelix spacing on both sides of the surfaces also vary. When in model $\mathrm{A}_{1}$ the surface stacking was only a function of $x$, it is now a function of $\alpha$ and $x$. Let us focus our attention on the hills of the sinusoidal surfaces: let $z_{0}$ be the initial molecular position, the $z$ coordinate of molecules at the hills of the sinusoidal surfaces is therefore $z=z_{0}+a_{\max }\left|\cos \left(z_{0}\right)\right|$. The derivative of this function is directly related to the intermolecular spacing at the hills of the sinusoidal deformation that varies with $\alpha$ and can thus be described as $d_{0} \times \mathrm{d} z / \mathrm{d} z_{0}$, where $d_{0}$ is the initial intermolecular distance. This model shows a periodic alternation of dilated and compressed areas at the hills of the sinusoidal deformation. Passing from dilated to compressed areas is a continuous process when $\alpha=0+n \pi$, but we have to dwell on that when $\alpha=\pi / 2+n \pi$, the theoretical interhelix spacing passes abruptly from its minimum to its maximum. We can therefore imagine that the structure does not exactly behave as in the model but that molecular rearrangements do occur in order to erase the discontinuity at $\alpha=\pi / 2+n \pi$, thus allowing continuous intermolecular distance variations. Note that symmetrical results are obtained at the valleys of the sinusoidal deformation.

4.2.2 Molecular paths. - As in model $\mathrm{A}_{1}$, line integrals transform into complex periodic space curves resembling those previously described (Sect. 4.1.2). Let us however mention that their amplitude is here modulated with $\alpha$, thus avoiding the rise of the discontinuity of model $\mathrm{A}_{1}$ at $\alpha=\pi / 2$ (see Fig. 6b).

4.2.3 Different section planes of the model. Geometrical analysis. - Using the nail drawing convention, we sketched the patterns drawn by the molecular orientations projected onto section planes parallel to $z 0 x$ (Fig. 12b), parallel to $0 z$ and oblique to $0 x$ and $0 y$ (Fig. 12c) and oblique to the three axes (Fig. 12d). These three section planes are respectively parallel to the cholesteric and distortion periodicity directions (Fig. 12b), oblique to the sinusoidal distortion periodicity direction and parallel to the cholesteric one (Fig. 12c) and oblique to both (Fig. 12d). Once again, we focus our attention on the most likely fracture planes, i.e. exclude those normal to the sample surface $y 0 z$.

We shall not here dwell on the occurrence of double twist, bend and splay, and only mention that they are similar to those of model $A_{1}$, but modulated with the amplitude of the sinusoidal distortion. We shall only note that opposite handedness double twist areas meet the densest ones (Fig. 12b).

We did not illustrate the section planes normal to $z 0 x$ that produce quasi periodic arched patterns, the arches themselves being dissymmetric, flattened or separated by wide unidirectional alignments of fibrils, for these patterns are very similar to those obtained for model $\mathrm{A}_{1}$ (Fig. 8).

4.2.4 Comparison with the micrographs. - Theoretical sections of figure 12 are compared to the waved patterns of plates II and III (Figs. 11a, b, $c_{2}, d_{2}$ ). In both cases, the molecules lying parallel or close to the fracture plane draw waved patterns that can appear normal (Figs. 11a, $c_{2}$ ) or oblique (Figs. $11 \mathrm{~b}, \mathrm{~d}_{2}$ ) to the cholesteric stratification. The observed and theoretical waves are strongly asymmetrically located within the layer. Note that the crenelled aspect of the undulations of plate II (emphasized in plate IIb) also arises on $c$ section planes. A perfect sinusoidal aspect of the undulations would be obtained only on fracture planes normal to the sample surface (Fig. 12b) and are therefore very unlikely. Moreover, the theoretical features do present undistorted semi-layers similar to those observed on the micrographs.

4.2.5 Occurrence of the waved patterns within the replicas. - We must now recall that waved patterns are not observed everywhere on the replicas. Does that mean that the cholesteric structure is not distorted everywhere ? Let us mention that the measured undulation 


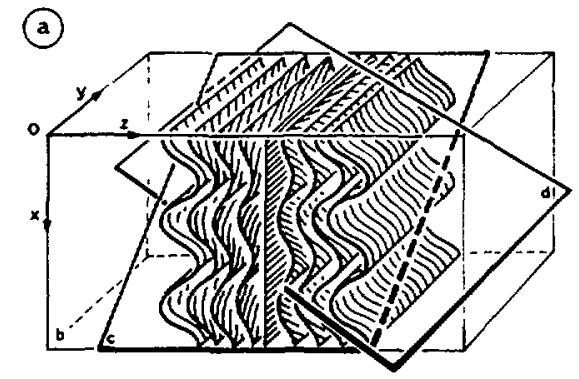

(b)

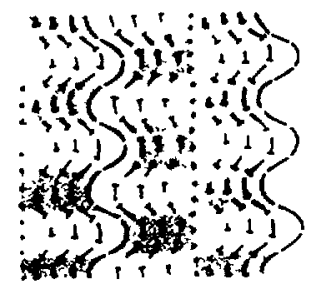

(c)

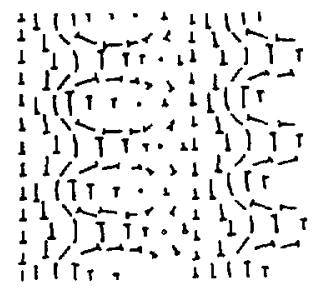

(d)

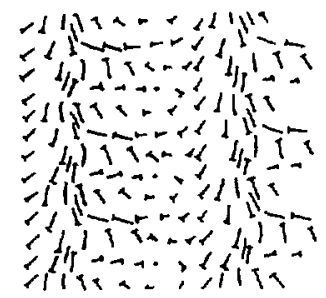

Fig. 12. - Model $A_{2}$. Theoretical patterns obtained by projection of the molecular orientations onto different section planes using the nail drawing convention : (b) section plane parallel to $x 0 z$ (frontview) : double twist of opposite handedness areas appear in grey, when those of double twist of same handedness are left white. These double twist areas are separated by single twist surfaces. Note that the first ones (grey tinted) meet areas of highest packing. (c) section plane parallel to $0 z$ and oblique to $0 x$ and $0 y$. (d) section plane oblique to the three axes.

periodicity $p_{\text {und }}^{\prime}$ varies with the relative orientations of the section plane and the undulation direction, i.e. with the obliquity of the fracture plane. Let $\beta$ be the angle of the fracture plane to the sample surface, then $p_{\text {und }}^{\prime}=p_{\text {und }} / \cos (\pi / 2-\beta)$ tends towards infinity when $\beta$ tends towards zero. As $\beta$ is generally small, $p_{\text {und }}^{\prime}$ may not be measurable since it is larger than the observation field, and the cholesteric layers hence appear undistorted. Table II summarizes the multiple kinds of features generated by this distortion of the cholesteric structure and their occurrence likelihood is compared with the experimental data. Let us mention that the 
Table II. - Recalls the multiple patterns that ought to rise from model $\mathrm{A}_{2}$. Their occurrence depends on the fracture plane. Let $\beta$ be the angle of the fracture plane to the sample surface. As the fracture is on average parallel to this latter, $\beta$ is usually small. We may therefore estimate the theoretical likelihood of the different patterns and compare it to the experimental data. The waved patterns do occur more frequently than they should do. This can be attributed to possible deviations from the theoretical orientation of the cholesteric layers.

\begin{tabular}{|c|c|c|c|c|}
\hline \multicolumn{2}{|c|}{ Patterns } & $\begin{array}{c}\text { Occurrence on the } \\
\text { replicas }\end{array}$ & \multicolumn{2}{c|}{ Theoretical likelihood } \\
\hline \multirow{3}{*}{ periodic } & $\begin{array}{c}\text { regular cholesteric } \\
\text { patterns }\end{array}$ & ++++ & ++++ & $\begin{array}{c}0<\beta<10^{\circ} \\
p_{\text {und }}^{\prime}>P_{\text {chol }}\end{array}$ \\
\cline { 2 - 5 } & periodic waves & ++ & + & $\begin{array}{c}\beta>10^{\circ} \\
p^{\prime} \text { und } \leq P_{\text {chol }}\end{array}$ \\
\hline \multirow{3}{*}{ quasi periodic } & $\begin{array}{c}\text { dissymmetric or } \\
\text { flattened arches }\end{array}$ & not determinable & ++ & $0<\beta<\pi / 2$-arctan(ak) \\
\cline { 2 - 5 } & sigmoidal arches & $\varepsilon$ & $\varepsilon$ & $\beta>\pi / 2$-arctan(ak) \\
\hline
\end{tabular}

probability of observation decreases with the increase of $\beta$. As experimental data fit the theoretical predictions, it is therefore possible to admit that the entire cholesteric phase is distorted.

The latter model therefore meets all the requirements to describe this undulation phenomenon, even though the real situation may not always correspond to a perfect normal orientation of the cholesteric layers relative to the sample surface. Besides, the higher frequency of waved patterns at the close vicinity of the isotropic phase may be due to a different orientation of the cholesteric layers at the isotropic interface, that would further the occurrence of the waved patterns.

This model nonetheless does not describe the additional features observed at the vicinity of the isotropic phase, i.e. the undulated arches and the strongly distorted patterns shown in plate IV.

4.3 MODEL B : SINUSOIDAL UNDULATION OF MOLECULAR ORIENTATIONS IN THE LAYERS (Fig. 3d). - Let us now recall the last theoretical kind of undulating modification of the cholesteric structure we introduced above, i.e. the sinusoidal undulation of the molecular paths within their plane (Fig. 3d). Any theoretical oblique section of such a structure reveals rows of undulated arches (not illustrated) that resemble those of plate IVa.

On the replicas, these undulated arches turn into the strongly distorted patterns of plate IVb as the orientation of the layers changes, when any theoretical section of such a structure reveals undulated arches whatever its orientation is. We therefore, and besides its theoretical unlikeliness, rule out this hypothesis. We may then put forward that the undulated arches correspond to a particular fracture plane of a more complex structure that in fact may certainly correspond to a combination of both phenomena, i.e. a sinusoidal undulation of the molecular paths within their planes combined to a sinusoidal distortion of the planes themselves. Note that if the two wavelengths are related (which is most likely) the molecular paths then follow helixlike curves. The projection of molecular orientations onto different section planes of such a complex helicoidal structure draws undulated arches as well as fairly complex patterns that may resemble that of plate $\mathrm{IVb}$. The analysis of this model will not be undertaken here. 


\section{Discussion.}

These models give a good description of the structural modifications of a cholesteric liquid crystalline sample subjected to a compressive stress applied parallel to the layers. The anisotropy of the response of the cholesteric structure to such a stress is in no way surprising. Moreover, the structural modifications seem to be modulated by the polymer concentration. In the more concentrated samples, the cholesteric planes are distorted into sinusoidal surfaces, when at lesser DNA concentrations a combination of two different kinds of sinusoidal deformation occurs : a sinusoidal undulation of molecular directors within the surfaces superimposes on the plane distortion, the molecular directors thus drawing helixlike curves. The combination of these two phenomena can correspond either to their simultaneous or to their sequential occurrence, the second one then corresponding to the relaxation of the first one. The absence of the second type of undulation in the concentrated samples would therefore be due either to poor molecular freedom or to a lower propagation speed of this process relative to the freezing rate, that would forbid the relaxation occurrence before the structure congeals. Whichever of these assumptions is kept, there would therefore be a molecular cohesiveness of these planes that had a priori no physical significance.

We deal here with structural data relating extremely swift dynamical phenomena and present their geometrical description. They may be compared to those described by Helfrich [19, 20], Scaramuzza et al. [21] and Carbone et al. [22] in cholesteric liquid crystals and Durand [23, 24] and Clark and Meyer [25] in smectic liquid crystals : between slide and coverslip, a lamellar liquid crystalline sample subjected to a dilative stress applied normally to its layers shows, above a critical strain threshold, long range sinusoidal undulations that do appear on a short time scale. Cholesteric liquid crystalline undulations were shown to occur within $10^{-1}$ to $10^{-2} \mathrm{~s}$ [22]. We estimated the freezing rate in our experiment after bibliographical data [26] that relate the cooling rate to the occurrence and size of ice crystals within a frozen hydrated sample : 1 to $2 \mu \mathrm{m}$ ice crystals form as cooling rate is in the $10^{3} \mathrm{Ks}^{-1}$ range, when vitrification (freezing of water in its amorphous state) is achieved for cooling rates of $10^{4} \mathrm{Ks}^{-1}$. Even at high magnification, we did not distinguish within our replicas (upper $10 \mu \mathrm{m}$ of the samples) any ice crystal ; their size, if ever some do form, is therefore smaller than the microscope resolution and the freezing rate may be estimated as some $10^{4} \mathrm{Ks}^{-1}$. The temperature of the sample therefore passes from 273 to $10 \mathrm{~K}$ within about $3 \times 10^{-2} \mathrm{~s}$. In a first approximation, we may then put forward that the structural modifications we observed here may correspond to a time scale of the same range as that mentioned above [22]. However, the given time for the occurrence of liquid crystal deformations may in fact be much less in our samples, since after some $5 \times 10^{-3} \mathrm{~s}$ the sample has already reached its solid state (the temperature is estimated as $243 \mathrm{~K}$ ). These structural deformations may therefore be quicker than those described in the literature [19-22]. This could explain in our experiments the undulation wavelength that is very small relative to the cholesteric pitch, when bibliographical data deal with long range undulation of the layers, the wavelength being then much larger than the cholesteric pitch.

Besides, we cannot rule out the occurrence of plastic deformations within the highly visquous or solid state when the temperature falls down to $10 \mathrm{~K}$. We dispose here of no data allowing to settle undoubtedly the origin of the observed deformations. However, we did never observe cracks or lacunae within the samples.

These structures can more generally be related to those of any geometrically or mechanically constrained cholesteric liquid crystal that yields structural instabilities. These instabilities raise -1 and +1 lines with no discontinuity of the molecular orientations, as well as bend and splay, that have been previously described by Lequeux [27]. Our experiments allow a molecular visualization of these phenomena. Similar local arrangements have also been shown 
within precholesteric organizations of long DNA molecules [28] and may also be related to the geometry of the blue phases.

We would like now to stress the biological interest of such a study. We give here a geometrical description of continuous deformations that have been experimentally induced by the application of a compressive stress. Let us now recall that cholesteric-like organizations of numerous biopolymers (DNA, collagen, chitin, cellulose) are widespread in nature [29] and involved in many morphogenetical processes. We think that the analysis of cholesteric continuous deformations may be of importance. Let us yet mention that sinusoidal distortion of a cholesteric analogue has already been observed by Lee [30] in the cuticle of a nematode (Mermis nigrescens), composed of collagen fibres. This distortion corresponds to the above described model $\mathrm{A}_{1}$ and may certainly describe the structural modification associated with the contraction of the animal body. Similar patterns are observed in plant cell walls made of cellulose [31] as well as in the cellulose matrix of the tunic of the ascidian Halocynthia papillosa [32]. The lack of amplitude modulation in the undulation of the cholesteric planes is certainly due to the presence of chemical links between molecules of successive planes that abolish the liquid properties and therefore modify the behaviour of the planes. Although we did not find any other bibliographical data dealing with such a phenomenon, we nevertheless think that it may be involved in many biological processes, morphogenetical as well as contraction processes.

\section{Acknowledgments.}

We would like to thank Pr. Yves Bouligand for fruitful discussions and a critical reading of the manuscript and Dr. Jean-Baptiste Fournier and Sigfrido Zayas for their kind contribution to mathematical formulations.

This work was supported by a grant from ARC and a grant from INSERM (91.0909).

Appendix (A. Leforestier).

Let $M(X, 0,0)$ be any point of the $0 x$ axis. The $x 0 y$ plane deformation into the sinusoidal surface $z=a \sin (k x)$ (where $a$ and $k$ are two constants) transforms $M$ into $M^{\prime}\left(X^{\prime}, 0, a \sin \left(k X^{\prime}\right)\right)$.

$$
\begin{aligned}
& X=\int_{0}^{x^{\prime}} \sqrt{1+a^{2} k^{2} \cos ^{2}(k x)} d x \\
& X=\sqrt{a^{2}+k^{-2}} E\left(\frac{k a}{\sqrt{a^{2} k^{2}+1}}, k X^{\prime}\right) \quad \text { with } \quad E(K, \Phi)=\int_{0}^{\Phi} \sqrt{1-K^{2} \sin ^{2} \phi} \mathrm{d} \phi .
\end{aligned}
$$

Then $X^{\prime}=k^{-1} E^{-1}\left(\frac{a}{\sqrt{a^{2}+k^{-2}}}, \frac{X}{\sqrt{a^{2}+k^{-2}}}\right)$.

Let us now consider any line $D_{0}$ of the $x 0 y$ plane and $\alpha$ the angle of $D_{0}$ to $0 x . D_{0}(x, x \tan \alpha, 0)$ therefore transforms into the space curves $S$ (see Fig. $5 c$ ) :

$$
\begin{aligned}
& S:\left(k^{-1} E^{-1}\left(\frac{a}{\sqrt{a^{2}+k^{-2}}}, \frac{x}{\sqrt{a^{2}+k^{-2}}}\right)\right. \\
& \left.\qquad x \tan \alpha, a \sin \left(E^{-1}\left(\frac{a}{\sqrt{a^{2}+k^{-2}}}, \frac{x}{\sqrt{a^{2}+k^{-2}}}\right)\right)\right)
\end{aligned}
$$


(a)

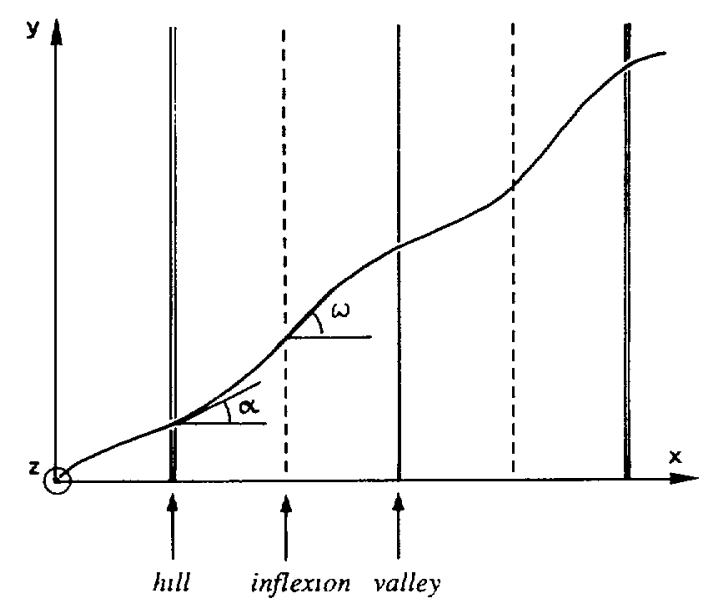

(b)

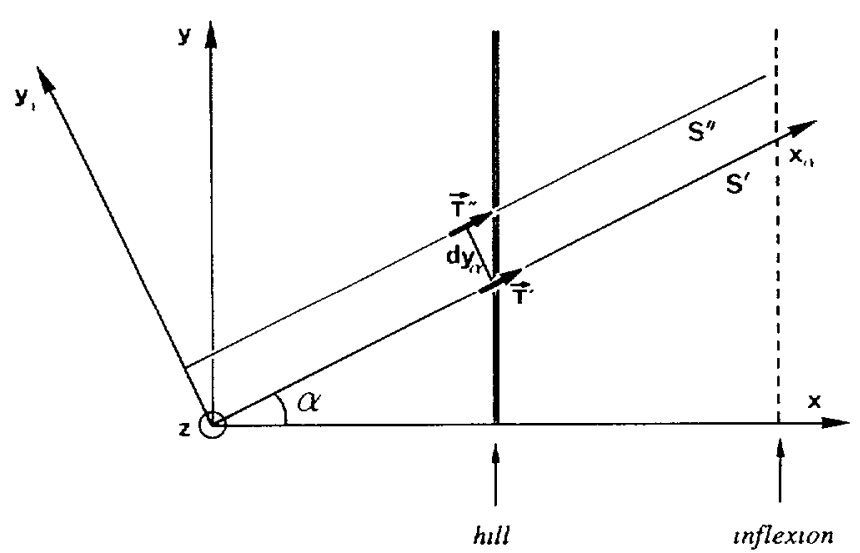

Fig. 13.

i.e.

$$
\begin{aligned}
& x=k^{-1} E^{-1}\left(\frac{a}{\sqrt{a^{2}+k^{-2}}}, \frac{y}{\tan \alpha \sqrt{a^{2}+k^{-2}}}\right) \\
& z=a \sin \left(E^{-1}\left(\frac{a}{\sqrt{a^{2}+k^{-2}}}, \frac{y}{\tan \alpha \sqrt{a^{2}+k^{-2}}}\right)\right) .
\end{aligned}
$$

The projection of $S$ onto the $x 0 y$ initial plane draws periodic undulating plane curves represented in figure $13 \mathrm{a}$, that are worth dwelling on. We consider the slope of their tangent :

$$
\frac{\mathrm{d} y}{\mathrm{~d} x}=\tan \alpha \sqrt{1+a^{2} k^{2} \cos ^{2} k x}
$$


that oscillates from $\tan \alpha$ at the hills and valleys of the sinusoidal surface, to $\tan \omega=\tan \alpha \sqrt{1+a^{2} k^{2}}$ at the inflexion lines. At the hills and valleys of the sinusoidal surface, the space curves can therefore be locally approximated to the sinusoid $S^{\prime}$ obtained by the intersection of the sinusoidal surface with the plane $y=x \tan \alpha$.

We shall henceforth take this point into account and focus our analysis of the twist variations at the hills and valleys of the sinusoidal distortion. Geometrical analysis showed the occurrence of double twist. We consider the twist components defined by Frank [18] and recall that $t_{1}$ is everywhere left handed [15] when $t_{2}$ is alternatively left and right handed with nil value at the inflexion lines.

Twist values depend on the intermolecular distance and the twist angle. We recall that in this model we have arbitrarily set the interhelix spacing at the hills and valleys of the sinusoidal surface as the initial interhelix spacing $d_{0}$. Besides, the molecular orientations at the hills and valleys is the same as in the undistorted cholesteric structure, as shown above. Then $t_{1}=t_{0}, t_{0}$ being the initial cholesteric twist. As regards $t_{2}$, it varies with $\alpha$. Let $T^{\prime}$ be the unit vector tangent to $S^{\prime}$ and $R_{\alpha}$ the Cartesian coordinate system $0 x_{\alpha} y_{\alpha} z$, where $x_{\alpha}$ is the projection of $S^{\prime}$ onto $x 0 y$ (see Fig. 13b). Let us consider $\mathbf{T}^{\prime}$ components in $R_{\alpha}$.

$$
\mathbf{T}^{\prime} \cdot \frac{1}{\sqrt{1+a^{2} k^{2} \cos ^{2} \alpha \cos ^{2}(k x)}}\left(\begin{array}{c}
1 \\
0 \\
a k \cos \alpha \cos (k x)
\end{array}\right) .
$$

Let us now consider the sinusoid $S^{\prime \prime}$, parallel to $S^{\prime}$ and $\mathrm{d} y_{\alpha}$ away from the latter. Let $\mathbf{T}^{\prime \prime}$ be the unit vector tangent to $S^{\prime \prime}$. Then the $\mathbf{T}^{\prime \prime}$ components in $R_{\alpha}$ are :

$$
\mathbf{T}^{\prime \prime} \cdot \frac{1}{\sqrt{1+a^{2} k^{2} \cos ^{2} \alpha \cos ^{2}(k(x+\mathrm{d} x))}}\left(\begin{array}{c}
1 \\
0 \\
a k \cos \alpha \cos (k(x+\mathrm{d} x))
\end{array}\right)
$$

with $\mathrm{d} x=\mathrm{d} y_{\alpha} \sin \alpha$ and $x=x_{\alpha} \cos \alpha$.

Let $\theta$ be the angle of $\mathbf{T}^{\prime}$ to $\mathbf{T}^{\prime \prime}$, then $t_{2}=-\frac{\sin \theta}{\mathrm{d} y_{\alpha}}$

We may assume that $\theta$ is small, i.e. $\theta<1^{\circ}$ then $\sin \theta \approx \theta \approx \tan \theta$.

$$
\begin{gathered}
\tan \theta=\frac{\mathbf{T}^{\prime} \wedge \mathbf{T}^{\prime \prime}}{\mathbf{T}^{\prime} \cdot \mathbf{T}^{\prime \prime}} \\
\tan \theta=\frac{a k \cos \alpha(\cos (k x)-\cos (k(x+\mathrm{d} x)))}{1+a^{2} k^{2} \cos ^{2} \alpha \cos (k x) \cos (k(x+\mathrm{d} x))} \\
\tan \theta=\frac{a k \cos \alpha \sin (k x) \sin (k \mathrm{~d} x)}{1+a^{2} k^{2} \cos ^{2} \alpha \cos (k x) \cos (k(x+\mathrm{d} x))}
\end{gathered}
$$

At the hills and valleys of the sinusoidal distortion, $\cos (k x)=0$.

Then

$$
\begin{gathered}
\tan \theta \approx a k^{2} \mathrm{~d} y_{\alpha} \cos \alpha \sin \alpha \sin (k x) \\
t_{2} \approx-\frac{\tan \theta}{\mathrm{d} y_{\alpha}} \approx-a k^{2} \cos \alpha \sin \alpha \sin (k x)
\end{gathered}
$$

at the hills and valleys.

We shall then distinguish the situation at the hills of the sinusoidal distortion from that at its valleys. 
At the hills of the sinusoidal surface where the curvature is extremal and negative, $\sin (k x)=1$.

Then

$$
\begin{gathered}
t_{2} \approx-a k^{2} \cos \alpha \sin \alpha \\
t_{2} \leqslant 0 \text { for } \alpha \in[0, \pi / 2] \\
t_{2} \geqslant 0 \text { for } \alpha \in[\pi / 2, \pi]
\end{gathered}
$$

At the valleys of the sinusoidal surface, the curvature is extremal and positive, and $\sin (k x)=-1$.

Then

$$
\begin{gathered}
t_{2} \approx a k^{2} \cos \alpha \sin \alpha \\
t_{2} \geqslant 0 \text { for } \alpha \in[0, \pi / 2] \\
t_{2} \leqslant 0 \text { for } \alpha \in[\pi / 2, \pi] .
\end{gathered}
$$

Moreover, at the hills and valleys, $t_{2}$ is nil for $\alpha \equiv 0$ [ $\left.\pi / 2\right]$ and extremal for $\alpha \equiv \pi / 4[\pi / 2]$. It passes continuously from positive to negative values. Note that the theoretical $t_{2}$ magnitude is the same whatever its handedness is. We may nevertheless think that the physical system tends to minimize right handed twist magnitude, for it corresponds to a fairly unfavorable arrangement of DNA molecules (right handed double helices).

We must recall that these results regard model $A_{1}$. Double twist occurrence is qualitatively

\begin{tabular}{|c|c|c|c|c|}
\hline \multicolumn{2}{|c|}{ curvature } & $<0$ & 0 & $>0$ \\
\hline \multirow{4}{*}{ sign of $t_{2}$} & $\alpha=0$ & 0 & 0 & 0 \\
\hline & $0<\alpha<\pi / 2$ & (left handed) & 0 & $\begin{array}{c}+ \\
\text { (right handed) }\end{array}$ \\
\hline & $\alpha=\pi / 2$ & 0 & 0 & 0 \\
\hline & $\pi / 2<\alpha<\pi$ & $\begin{array}{c}+ \\
\text { (right handed) } \\
\end{array}$ & 0 & (left handed) \\
\hline \multicolumn{2}{|c|}{ sign of $t_{1}$} & \multicolumn{3}{|c|}{ - (left handed) ; independent of the sign of the curvature } \\
\hline
\end{tabular}
the same in model $A_{2}$ but is modulated with the amplitude variations.

These results thus do tally our geometrical analysis; they can be summarized by the table III that recalls that this structure consists of a periodic mosaic of domains in which the double twist is alternatively of same and opposite handedness.

Table III.

\section{References}

[1] Lydon J. E. and Robinson D. G., Biochim. Biophys. Acta 260 (1972) 298-311.

[2] Kleman M., Williams C. E., Costello M. J. and Gulik-Krzywicki T., Philos. Mag. 35 (1977) 33-56.

[3] Costello M. J., Meiboom S. and Sammon M. J., Phys. Rev. A 29 (1984) 2957-2959.

[4] Zazadzinski J. A. N., Meiboom S., Sammon M. J. and Berreman D. W., Phys. Rev. Lett. 57 (1986) 364-367. 
[5] Livolant F. and Bouligand Y., Mol. Cryst. Liq. Cryst. 166 (1986) 91-100.

[6] Goodman J. F. and Clunie J. S., Liquid crystals and plastic crystals 2 (Gray \& Winsor Eds., Ellis Horwood, 1974) pp. 1-23.

[7] Volgt-Martin I. G., Durst H., Reck B. and Ringsdorf H., Macromolecules 21 (1988) 16201626.

[8] Livolant F., Levelut A. M., Doucet J. and Benoit J. P., Nature 339 (1989) 724-726.

[9] Rill R. L., Livolant F., Aldrich H. C. and Davidson M. W., Chromosoma 98 (1989) 280-286.

[10] Voigt-Martin I. G., Krug H. and van Dyck D., J. Phys. France 51 (1990) 2347-2371.

[11] Zazadzinski J. A. N., J. Phys. France 51 (1990) 747-756.

[12] Livolant F., J. Mol. Biol. 218 (1991) 165-181.

[13] Leforestier A. and Livolant F., Biol. Cell. 71 (1991) 115-122.

[14] Strzelecka T. E. and Rill R. L., J. Am. Chem. Soc. 109 (1987) 4513-4518.

[15] Livolant F. and Maestre M. F., Biochemistry 27 (1988) 3056-3068.

[16] Gaill F. and Mosseri R., C.R. Acad. Sc. (Paris) 311 (1990) 1479-1485.

[17] FRANK F. C., Configurations and interactions of macromolecules and liquid crystals, Discussions of the Faraday Society, F. C. Tompkins Ed. 25 (The Aberdeen University Press Ltd., 1958) pp. 19-28.

[18] Bouligand Y., J. Phys. 35 (1974) 215-235.

[19] Helfrich W., Appl. Phys. Lett. 17 (1970) 531.

[20] Helfrich W., J. Chem. Phys. 55 (1971) 839.

[21] Scaramuzza N., Bartolino R. and Barbero G., J. Appl. Phys. 53 (1982) 8593-8598.

[22] Carbone V., Scaramuzza N. and Guarracino N., Mol. Cryst. Liq. Cryst. 188 (1990) 57-65.

[23] Durand G., C.R. Acad. Sc. (Paris) 275 (1972) 629-631.

[24] Ribotta R. and Durand G., J. Phys. France 38 (1977) 179-204.

[25] Clark N. A. and Meyer R. B., Appl. Phys. Lett. 22 (1973) 493.

[26] Robards A. W. and Sleytr U. B., Low Temperature Methods in Biological Electron Microscopy, Practical Methods in Electron Microscopy, A. M. Glauert Ed. 10 (Elsevier, 1985) pp. 5-130.

[27] LEQUEUX F., Quelques instabilités élastiques locales dans les cholestériques. Doctoral thesis, Orsay University (1988).

[28] Livolant F., J. Phys. France 48 (1987) 1051-1066.

[29] Bouligand Y., Liquid crystalline order in polymers. A. Blumstein Ed. (Academic Press, 1978) pp. 261-297.

[30] LEE D. L., J. Zool. Lond. 161 (1970) 513-518.

[31] Reis D., Vian B., Chanzy H. and Roland J. C., Biol. Cell. 73 (1991) 173-178.

[32] Gaill F., Private communication. 